\title{
Functional analysis of LHCSR1, a protein catalyzing NPQ in mosses, by heterologous expression in Arabidopsis thaliana
}

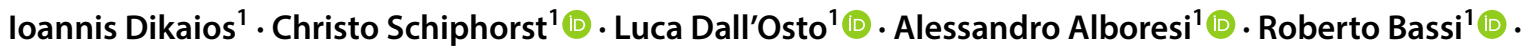 \\ Alberta Pinnola ${ }^{1,2}$ (1)
}

Received: 11 February 2019 / Accepted: 27 June 2019 / Published online: 3 July 2019

(c) The Author(s) 2019

\begin{abstract}
Non-photochemical quenching, NPQ, of chlorophyll fluorescence regulates the heat dissipation of chlorophyll excited states and determines the efficiency of the oxygenic photosynthetic systems. NPQ is regulated by a pH-sensing protein, responding to the chloroplast lumen acidification induced by excess light, coupled to an actuator, a chlorophyll/xanthophyll subunit where quenching reactions are catalyzed. In plants, the sensor is PSBS, while the two pigment-binding proteins Lhcb4 (also known as CP29) and LHCII are the actuators. In algae and mosses, stress-related light-harvesting proteins (LHCSR) comprise both functions of sensor and actuator within a single subunit. Here, we report on expressing the lhcsrl gene from the moss Physcomitrella patens into several Arabidopsis thaliana npq4 mutants lacking the $\mathrm{pH}$ sensing PSBS protein essential for NPQ activity. The heterologous protein LHCSR 1 accumulates in thylakoids of $A$. thaliana and NPQ activity can be partially restored. Complementation of double mutants lacking, besides PSBS, specific xanthophylls, allowed analyzing chromophore requirement for LHCSR-dependent quenching activity. We show that the partial recovery of NPQ is mostly due to the lower levels of Zeaxanthin in A. thaliana in comparison to P. patens. Complemented npq2npq4 mutants, lacking besides PSBS, Zeaxanthin Epoxidase, showed an NPQ recovery of up to $70 \%$ in comparison to A. thaliana wild type. Furthermore, we show that Lutein is not essential for the folding nor for the quenching activity of LHCSR1. In short, we have developed a system to study the function of LHCSR proteins using heterologous expression in a variety of A. thaliana mutants.
\end{abstract}

Keywords LHCSR $\cdot$ NPQ $\cdot$ Photoprotection $\cdot$ Heterologous expression $\cdot$ P. patens $\cdot$ A. thaliana

\section{Introduction}

The need for a balance between light harvesting and photoprotection is one of the key driving forces that shaped adaptation of photosynthetic eukaryotic organisms on Earth (Genty et al. 1990; Müller et al. 2001; Baker 2008). Non-photochemical quenching (NPQ) of chlorophyll (Chl) fluorescence acts through modulating the dissipation of $\mathrm{Chl}$ excited states into heat and balances the efficiency of the photosynthetic systems versus the electron transport rate,

Ioannis Dikaios and Christo Schiphorst contributed equally.

Electronic supplementary material The online version of this article (https://doi.org/10.1007/s11120-019-00656-3) contains supplementary material, which is available to authorized users.

Alberta Pinnola

alberta.pinnola@unipv.it

Extended author information available on the last page of the article thus avoiding photo-oxidative stress and photoinhibition due to excess light. NPQ includes components with different induction and relaxation kinetics: the fastest (1-2 min) and rapidly reversible type, $\mathrm{qE}$, depends on a trans-thylakoid $\Delta \mathrm{pH}$ promoted by excess light (Horton et al. 1996; Kramer et al. 1999; Kanazawa and Kramer 2002) which protonates specific residues on $\mathrm{pH}$ sensitive trigger proteins ( $\mathrm{Li}$ et al. 2004; Ballottari et al. 2016); qZ, is activated in 8-10 min and also depends on low luminal $\mathrm{pH}$ through the activation of violaxanthin (Vio) de-epoxidase (VDE), a lumenal enzyme-converting zeaxanthin (Zea) from pre-existing Vio. The slowest component, called qI, for photoInhibitory quenching, comprises components from the slow and reversible inactivation of Photosystem II (PSII) reaction centers as well as other long-term processes involved in acclimation to the light environment (Brooks et al. 2013). In some organisms, such as Chlamydomonas reinhardtii an additional component, qT, is due to the displacement of LHCII from PSII to PSI upon phosphorylation (Allorent et al. 2013). qE 
activation depends on a sensor for lumenal $\mathrm{pH}$, induced by excess light, coupled to a $\mathrm{Chl} / \mathrm{xanthophyll}$ actuator subunit where quenching reactions are catalyzed upon the establishment of specific pigment-pigment interactions (Allorent et al. 2013). The protein PSBS is a typical $\mathrm{pH}$ sensor ( $\mathrm{Li}$ et al. 2000) which does not bind chromophores (Dominici et al. 2002; Fan et al. 2015), but is able to activate quenching within the interacting antenna protein subunits Lhcb4 (CP29) (Ahn et al. 2008; de Bianchi et al. 2011) and LHCII (Ruban et al. 2007; Dall'Osto et al. 2017). In the case of algae the trigger of $\mathrm{qE}$ is LHCSR, a pigment binding protein (Peers et al. 2009) which also hosts protonatable residues (Liguori et al. 2013; Ballottari et al. 2016) thus comprising both sensing and catalytic functions in a single subunit (Bonente et al. 2011). The moss Physcomitrella patens, a descendant from an evolutionary intermediate between algae and plants, hosts both PSBS and LHCSR each active in $\mathrm{qE}$ (Alboresi et al. 2010; Gerotto et al. 2012) which suggests that LHCSR might be active in vascular plants. Modification of $\mathrm{qE}$, timescales in which PSBS and LHCSR1 are active, can improve crop productivity depending on the growth conditions (Horton 2000). Since the NPQ activity of PSBS and LHCSR 1 are cumulative in P. patens and LHCSR 1 is not present in plants, re-introducing LHCSR1 in vascular plants could enhance the dynamic range of NPQ with positive effects on crop productivity. In this work, we used the $n p q 4$ mutant of $A$. thaliana, lacking PSBS and, therefore $\mathrm{qE}$, as a host for the expression of $P$. patens LHCSR1. We proceeded to verify the possibility of expressing LHCSR 1 in vascular plants and its capability to complement the NPQ function in genotypes lacking PSBS. The availability of a large library of $A$. thaliana mutants affected in energy dissipation makes transformation by Agrobacterium mediated floral dipping (Clough and Bent 1998), an efficient tool for elucidation of the quenching mechanism in LHCSR1. As a proof of concept, we determined the requirement of specific xanthophyll co-factors for LHCSR1-dependent quenching.

\section{Materials and methods}

\section{Cloning of LHCSR1 CDNA, A. thaliana transformation and screening}

The fragment corresponding to LHCSRl (Locus XM_024529130) was amplified from $P$. patens total cDNA obtained from 6-days-old plants grown on minimal medium, RNA was isolated using TRI Reagent ${ }^{\circledR}$ Protocol (T9424, Sigma-Aldrich) and cDNA was synthetized using M-MLV Reverse Transcriptase (M1302, Sigma-Aldrich) and Oligo(dT) $)_{23}(\mathrm{O} 4387$, Sigma-Aldrich). Primers including $a t t B$ sequences for the gateway technology (Invitrogen ${ }^{\mathrm{TM}}$ ) were designed to anneal 27 base pairs upstream of the ATG codon (PpLHCSR1attB1 5'-GGGGACAAGTTTGTACAA AAAAGCAGGCTCCAATCTCGAGCTTTTGCT-3') and 107 base pairs downstream of the stop codon (PpLHCSR1attB 5'- GGGGACCACTTTGTACAAGAAAGCTGG GTCGACTGCGAATCAATCAGAA-3'). The PCR-product was first cloned in pDONR ${ }^{\mathrm{TM}} 221$ Vector (12536-017, Invitrogen $^{\mathrm{TM}}$ ) and then recombined into the $\mathrm{pH} 7 \mathrm{WG} 2$ binary vector (Karimi et al. 2002) to make the 35 S::lhcsrl construct. The accuracy of the cloning was verified by DNA digestion and sequencing and the plasmid was transferred to Agrobacterium tumefaciens strain GV3101 (Zhang et al. 2006). A. thaliana plants were transformed by the floral dip method and transgenic plants were selected on MurashigeSkoog medium supplemented by hygromycin $\left(25 \mathrm{mg} \mathrm{L}^{-1}\right)$ and carbenicillin (100 $\mathrm{m} \mathrm{L}^{-1}$ ) (Clough and Bent 1998).

\section{Plant material and growth conditions}

Physcomitrella patens protonema tissue was grown in petri dishes containing minimum $\mathrm{PPNO}_{3}$ medium (Ashton et al. 1979) enriched with $0.5 \%$ glucose and solidified with $0.8 \%$ plant agar. Material was grown under controlled light and temperature conditions: $24{ }^{\circ} \mathrm{C}, 16$-h light/8-h dark photoperiod with a light intensity of $60 \mu \mathrm{mol}$ photons $\mathrm{m}^{-2} \mathrm{~s}^{-1}$. A. thaliana plants (ecotype Columbia) were grown in controlled conditions of 8-h light/16-h dark with a light intensity of $100 \mu \mathrm{mol}$ photons $\mathrm{m}^{-2} \mathrm{~s}^{-1}$ under stable temperature $\left(23{ }^{\circ} \mathrm{C}\right.$ in light $/ 20^{\circ} \mathrm{C}$ in dark $)$.

\section{Gel electrophoresis}

Total leaf extracts from transgenic A. thaliana plants were homogenized using plastic pestles in Laemmli buffer with $62.5 \mathrm{mM}$ Tris pH 6.8, 10\% glycerol, 5\% SDS, 5\% $\beta$-mercaptoethanol and loaded on a $15 \%$ (w/v) separating acrylamide gel (75:1 acrylamide/bis-acrylamide) with $6 \mathrm{M}$ Urea. After SDS-PAGE gel electrophoresis, proteins were transferred by western blot on a polyvinylidene fluoride (PVDF) transfer membrane (Millipore) with the use of a Bio-rad blot system and developed using specific LHCSR and CP43 or CP47 antibodies produced in the laboratory.

\section{Thylakoid isolation and thylakoid fractionation}

Thylakoids were purified from about 25 days old $A$. thaliana WT and transgenic plants (Berthold et al. 1981). Detached leaves from dark-adapted plants were harvested and homogenized in cold extraction buffer containing $0.02 \mathrm{M}$ Tricine$\mathrm{KOH}$ pH 7.8, $0.4 \mathrm{M} \mathrm{NaCl}, 0.002 \mathrm{M} \mathrm{MgCl}_{2}, 0.5 \%$ milk powder, and protease inhibitors $5 \mathrm{mM} \varepsilon$-aminocaproic acid, $1 \mathrm{mM}$ phenyl-methylsulfonyl fluoride and $1 \mathrm{mM}$ benzamidine added right before the isolation. Homogenized leaves were then filtered, centrifuged at $1500 \times g$ for $15 \mathrm{~min}$ at $4{ }^{\circ} \mathrm{C}$ 
and then resuspended in a hypotonic buffer of $20 \mathrm{mM}$ Tricine- $\mathrm{KOH} \mathrm{pH} 7.8,5 \mathrm{mM} \mathrm{MgCl} 2,150 \mathrm{mM} \mathrm{NaCl}$ and the pre-mentioned concentrations of protease inhibitors. Resuspended thylakoids were centrifuged for $10 \mathrm{~min}$ at $10,000 \times \mathrm{g}$ $\left(4{ }^{\circ} \mathrm{C}\right)$ followed by a second resuspension in a sorbitol buffer (10 mM HEPES-KOH pH 7.5, 0.4 M Sorbitol, $15 \mathrm{mM} \mathrm{NaCl}$ and $5 \mathrm{mM} \mathrm{MgCl}$ ). Thylakoid membranes were quantified and either used directly or stored in $-80{ }^{\circ} \mathrm{C}$.

Solubilization was performed as in (Morosinotto et al. 2010; Pinnola et al. 2015a, b). Isolated thylakoids were resuspended in $20 \mathrm{mM}$ HEPES-KOH, $\mathrm{pH} 7.5,15 \mathrm{mM} \mathrm{NaCl}$, $5 \mathrm{mM} \mathrm{MgCl}{ }_{2}$ buffer at $1 \mathrm{mg} \mathrm{Chl} / \mathrm{mL}$ and solubilized at $4{ }^{\circ} \mathrm{C}$ for $20 \mathrm{~min}$ in slow agitation with different amounts of $\alpha$-DM ranging from 0.16 to $0.49 \%(\mathrm{w} / \mathrm{v})$, always in the presence of $15 \mathrm{mM} \mathrm{NaCl}, 5 \mathrm{mM} \mathrm{MgCl}$ and $10 \mathrm{mM}$ HEPES-KOH, $\mathrm{pH}$ 7.5. Unsolubilized thylakoids were pelleted by centrifugation at $3500 \times g$ for 5 min. Partially solubilized grana membranes were instead pelleted with a further $30 \mathrm{~min}$ centrifugation at $40,000 \times g$. Solubilized complexes and small membrane patches remained in the supernatant. Membrane pellet was washed with $15 \mathrm{mM} \mathrm{NaCl}, 5 \mathrm{mM} \mathrm{MgCl}_{2}$ and $20 \mathrm{mM}$ HEPES-KOH, $\mathrm{pH} 7.5$, centrifuged for $30 \mathrm{~min}$ at $30,000 \times g$ and finally resuspended in $0.4 \mathrm{M}$ Sorbitol, $10 \mathrm{mM}$ HEPES-KOH, pH 7.5, $15 \mathrm{mM} \mathrm{NaCl}, 5 \mathrm{mM} \mathrm{MgCl}_{2}$ frozen in liquid nitrogen and stored at $-80^{\circ} \mathrm{C}$ until use.

\section{Pigment-protein complexes separation with Deriphat-PAGE}

Non-denaturating Deriphat-PAGE was performed as previously described (Peter et al. 1991) with some modifications: stacking gel of 3.5\% (w/v) acrylamide (38:2 acrylamide/bisacrylamide) and separating acrylamide gel was prepared at different fixed or gradient concentration depending on the purposes. Acrylamide concentrations are specified along the text. Thylakoids from wild type and transgenic plants corresponding to a final $\mathrm{Chl}$ concentration of $0.5 \mathrm{mg}$ were washed with $5 \mathrm{mM}$ EDTA and then resuspended in $10 \mathrm{mM}$ HEPES pH 7.5. Samples were then solubilized with $0.8 \%$ n-Dodecyl $\alpha$-D-maltoside and $10 \mathrm{mM}$ HEPES $\mathrm{pH} 7.5$ by vortexing thoroughly for $1 \mathrm{~min}$. Solubilized samples were kept $10 \mathrm{~min}$ in ice and then centrifuged at $15,000 \times \mathrm{g}$ for $10 \mathrm{~min}$ to pellet any insolubilized material and then loaded.

\section{Fluorescence measurements}

In vivo Chl fluorescence was measured at room temperature after leaves were dark adapted for $45 \mathrm{~min}$, by FC $800 \mathrm{MF}$ closed FluorCam Video-imaging system (Photon Systems Instruments, Czech Rep.) and PAM-100 (Walz, Germany) fluorometers. For every measurement a saturating pulse of $4000 \mu \mathrm{mol}$ photons $\mathrm{m}^{-2} \mathrm{~s}^{-1}$ and actinic light with an intensity of $1200 \mu \mathrm{mol}$ photons $\mathrm{m}^{-2} \mathrm{~s}^{-1}$ were applied. $\mathrm{F}_{\mathrm{v}} / \mathrm{F}_{\mathrm{m}}$ and NPQ parameters were calculated as $\left(F_{\mathrm{m}}-F_{\mathrm{o}}\right) / F_{\mathrm{m}}$ and $\left(F_{\mathrm{m}}-F_{\mathrm{m}}{ }^{\prime}\right) / F_{\mathrm{m}}{ }^{\prime}$ respectively.

\section{Pigment composition analysis (HPLC)}

Arabidopsis thaliana leaves or $P$. patens protonema tissue was frozen in liquid nitrogen and homogenized using plastic pestles. Pigments were extracted in $80 \%$ ice-cold acetone (buffered with $\mathrm{NaHCO}_{3}$ ) and analyzed by high-pressure liquid chromatography (HPLC) after a twostep centrifugation at $21,000 \times g$ for $10 \mathrm{~min}$ at $4{ }^{\circ} \mathrm{C}$.

\section{9-aminoacridin measurements}

Intact chloroplasts were isolated from 4- to 5- week old $A$. thaliana (npq4 + LHCSR1) plants or 6-days-old P. patens psbs-lhcsr 2 ko $\left(\mathrm{PzL}_{2}\right)$ based on the method from (Munekage et al. 2002). Tissue was homogenized using a potter in ice-cold buffer containing $330 \mathrm{mM}$ sorbitol, $20 \mathrm{mM}$ Tricine/ $\mathrm{NaOH}$ (pH 7.6), $5 \mathrm{mM}$ EDTA, 5 mM EGTA, $10 \mathrm{mM}$ $\mathrm{NaHCO}_{3}, 5 \mathrm{mM} \mathrm{MgCl}, 0.1 \%$ (w/v) BSA and $1.87 \mathrm{mM}$ Sodium L-ascorbate. The homogenized tissue was filtered through a nylon mesh and the filtrate was centrifuged at $2000 \times g$ for $5 \mathrm{~min}$ in a pre-chilled centrifuge $\left(4^{\circ} \mathrm{C}\right)$. The pellet was resuspended in an ice-cold buffer containing $300 \mathrm{mM}$ Sorbitol, $10 \mathrm{mM}$ HEPES/NaOH (pH 7.6), $5 \mathrm{mM} \mathrm{MgCl}_{2}$, $10 \mathrm{mM} \mathrm{NaHCO}, 2.5 \mathrm{mM}$ EDTA and $1.87 \mathrm{mM}$ of Sodium L-ascorbate. Samples were kept on ice until right before the measurements. Chloroplasts were diluted to $25 \mu \mathrm{g} / \mathrm{mL} \mathrm{Chl}$ in a buffer at room temperature containing $50 \mathrm{mM}$ Tricine/ $\mathrm{NaOH}$ (pH 8.0), $100 \mathrm{mM} \mathrm{NaCl}, 10 \mathrm{mM} \mathrm{MgCl}_{2}$ and 9-aminoacridine $(2 \mu \mathrm{M})$ and measurements were performed at different light intensities 50, 200, 500 and $800 \mu \mathrm{mol}$ photons $\mathrm{m}^{-2} \mathrm{~s}^{-1}$. Fluorescence measurements were recorded on a Fluoromax-3 (Horiba scientific), excitation wavelength $400 \mathrm{~nm}$, emission measured at $430 \mathrm{~nm}$. Electron transport was induced using the actinic light of a pulse-amplitude modulated fluorimeter (Heinz-Walz) equipped with a red filter $(600-750 \mathrm{~nm}), 40 \mathrm{~s}$ of dark adaptation, $80 \mathrm{~s}$ illumination and $40 \mathrm{~s}$ of recovery in the dark.

\section{Results}

\section{LHCSR1 expression in A. thaliana npq4 mutant}

The coding sequence of LHCSR 1 was amplified from cDNA synthetized from $P$. patens protonema, cloned in the $\mathrm{pH} 7 \mathrm{WG} 2$ vector under the control of the constitutive $35 \mathrm{~S}$ promoter and used for Agrobacterium-mediated transformation of $n p q 4$ mutant plants. The $n p q 4$ mutants are devoid of $\mathrm{qE}$ due to the absence of PSBS. Transgenic seeds were collected and grown on hygromycin-B, resistant seedlings 
were transferred to soil, together with A. thaliana wild type (WT) and npq4 control plants. Leaf extracts from A. thaliana genotypes and $P$. patens protonema tissue were analyzed by western blotting using $\alpha$-LHCSR (Pinnola et al. 2013) and $\alpha$-CP43 antibodies. WT and $n p q 4$ plants showed no reaction with $\alpha$-LHCSR while CP43 was detected in all samples (Fig. S1b). In P. patens both LHCSR1 and LHCSR2 were detected. In A. thaliana a single band, corresponding to LHCSR1, was obtained in hygromycin-resistant $A$. thaliana plants with a mobility matching the native LHCSR1 protein in $P$. patens thylakoid membranes. This suggests that $P$. patens LHCSR 1 is both expressed and processed to its mature form in A. thaliana. The strongest LHCSR1 expressors among the complemented A. thaliana npq4 lines were selected (C1, C3 and A5) and used to create homozygous lines, these lines contained multiple insertions which was established from the segregation pattern in later generations. It was verified that the $\mathrm{T} 4$ generation of line $\mathrm{C} 1$ was stable and further experiments, unless otherwise indicated, were performed on the $\mathrm{T} 5$ generation of this line. A quantitative western blot showed that these plants contain $82.8 \pm 1.8 \%$ of LHCSR1 in comparison to the LHCSR1-only P. patens psbs-lhcsr2 knock-out (ko) (Fig. S2).

\section{LHCSR1 localization in A. thaliana thylakoid membranes}

Thylakoid membranes from A. thaliana npq4 plants expressing LHCSR 1 and control $n p q 4$ plants were purified and analyzed by SDS-PAGE (Fig. 1a). A band with the apparent molecular weight of LHCSR1 was present with a mobility between Lhcb3 and Lhcb6 (CP24) in the complemented plants but not in the background line $n p q 4$. The new band from the LHCSR 1 expressing A. thaliana thylakoids was excised from gel and submitted to mass spectrometric analysis which yielded 10 peptides covering $58 \%$ of the mature protein sequence (Fig. S3). Each peptide matched the theoretical mass calculated from the DNA sequence, as predicted by ChloroP (Emanuelsson et al. 1999), implying no post-translational modifications were present within the identified fragments. No other identifiable changes in protein composition could be detected between the two genotypes (Fig. 1a). Furthermore, western blot analysis confirmed that the $\alpha$-LHCSR antibody reacted against the LHCSR 1 protein accumulated in the thylakoid membranes of the complemented plants (Fig. 1b) with the same electrophoretic mobility as the native protein from $P$. patens. LHCSR 1 and LHCSR 2 were detected in the WT $P$. patens thylakoids, but not in the $P$. patens lhcsrl-lhcsr2 ko thylakoid membranes (Fig. 1b).

The distribution of LHCSR 1 in the thylakoid domains of A. thaliana, was assessed by thylakoid fractionation with n-dodecyl $\alpha$-D-maltoside ( $\alpha$-DM) (Morosinotto et al. 2010;
Pinnola et al. 2015a, b). The procedure yielded a pellet enriched in grana membranes and a supernatant comprising the stroma lamellae. The Coomassie-stained SDS-PAGE gel confirmed PSI and ATPase were in the stroma-derived supernatant fraction while the PSII core subunit as well as LHCII and Lhcb6 (CP24) were enriched in the pellet, i.e. the grana partitions (Fig. 1c). A band with the apparent molecular weight corresponding to LHCSR 1 was highly enriched in the supernatant, suggesting that recombinant LHCSR 1 was localized in thylakoid stroma-exposed membranes of complemented A. thaliana npq4. This was confirmed by western blot (Fig. 1d) and step-solubilization with increasing concentrations of $\alpha$-DM detergent, namely $0.16,0.24,0.32$, 0.39 and $0.47 \% \alpha$-DM (Fig. S4). Immunoblotting showed that LHCSR 1 was already enriched in the stromal fraction at $0.16 \% \alpha$-DM with a $\mathrm{Chl} a / b$ ratio $>6.0$ and a polypeptide composition, including PSI and ATPase, typical of stroma membranes (Fig. S4a, b). Low amounts of LHCSR1 were found in the pellet fractions up to $0.32 \% \alpha$-DM suggesting that the protein might also be localized in the margins of $A$. thaliana thylakoids.

\section{Pigment binding to recombinant LHCSR1 and their spectra}

LHCSR is a pigment-binding protein in $C$. reinhardtii and $P$. patens (Bonente et al. 2011; Pinnola et al. 2013). To verify that recombinant LHCSR1 expressed in A. thaliana did actually refold properly with pigments, thylakoid membranes were analyzed by Deriphat PAGE (Fig. 2a). Although the protein composition was similar between the two genotypes, A. thaliana $n p q 4+$ LHCSR 1 did contain two additional bands with respect to A. thaliana $n p q 4$, migrating, respectively, just below the monomeric LHC band and in between LHC monomers and trimers. Gel slices were excised from the gel and further separated by denaturing SDS-PAGE, followed by western blotting. The two "additional" bands in the gel from A. thaliana npq4 + LHCSR1 showed strong reaction towards the $\alpha$-LHCSR antibody (Fig. 2b). Fainter reactions were also obtained with fractions from in between the two bands but not with those at lower or higher mobility, suggesting that LHCSR1 migrated initially as a dimeric Chl binding protein which partially dissociated into monomers during solubilization and/or electrophoretic migration.

LHCSR proteins have a characteristically red-shifted absorption spectrum with respect to other LHC proteins (Bonente et al. 2011; Pinnola et al. 2013, 2015b). Absorption spectra recorded from extracted gel bands showed a red-shifted $Q_{\mathrm{y}}$ peak at $679.1 \mathrm{~nm}$ with respect to LHCII trimers $(674.2 \mathrm{~nm})$ and LHC monomers $(676.9 \mathrm{~nm})$, typical for LHCSR1 (Bonente et al. 2011; Pinnola et al. 2017) (Fig. 2c). Also, the LHCSR 1-containing band was depleted of $\mathrm{Chl} b$ with respect to the bands from other LHCs, thus copying the 
A

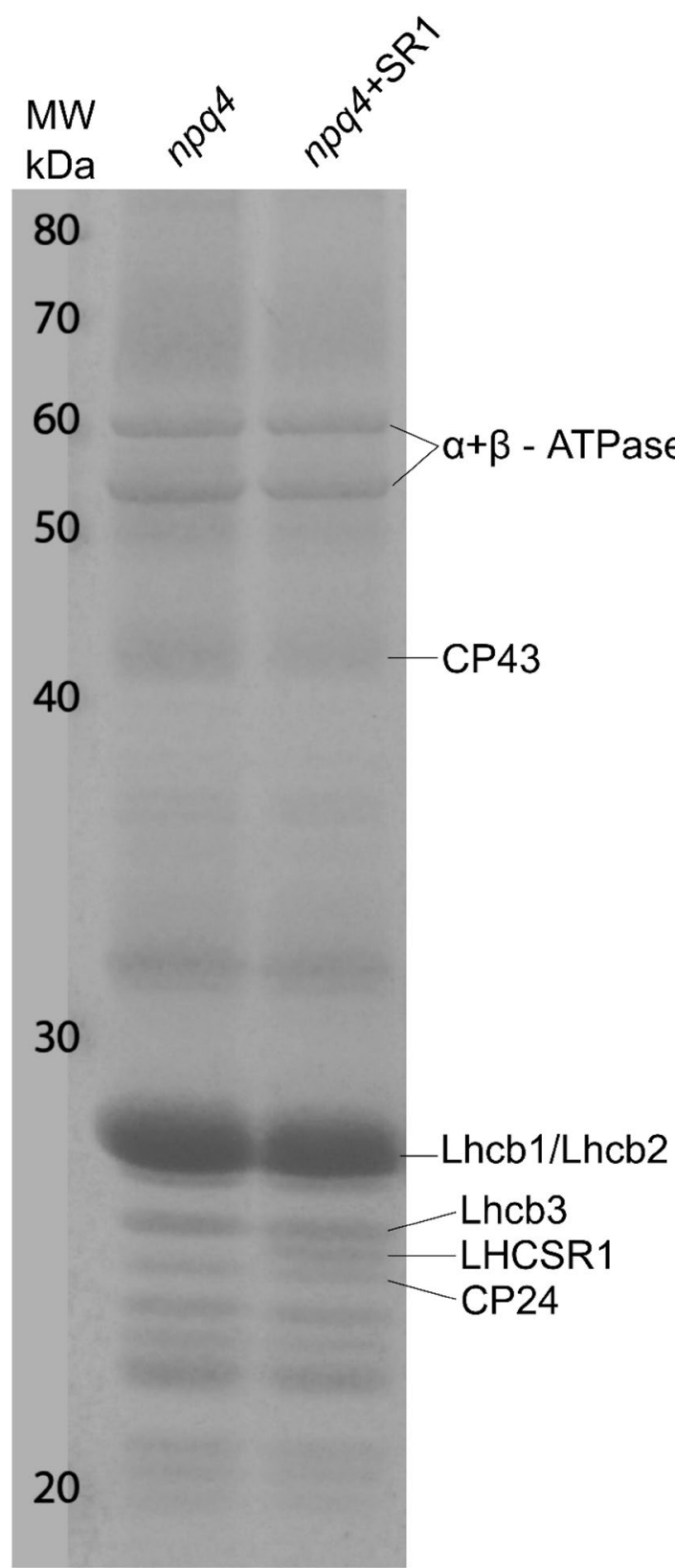

B

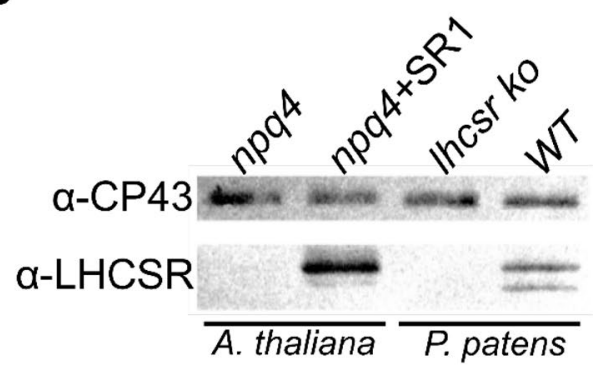

C

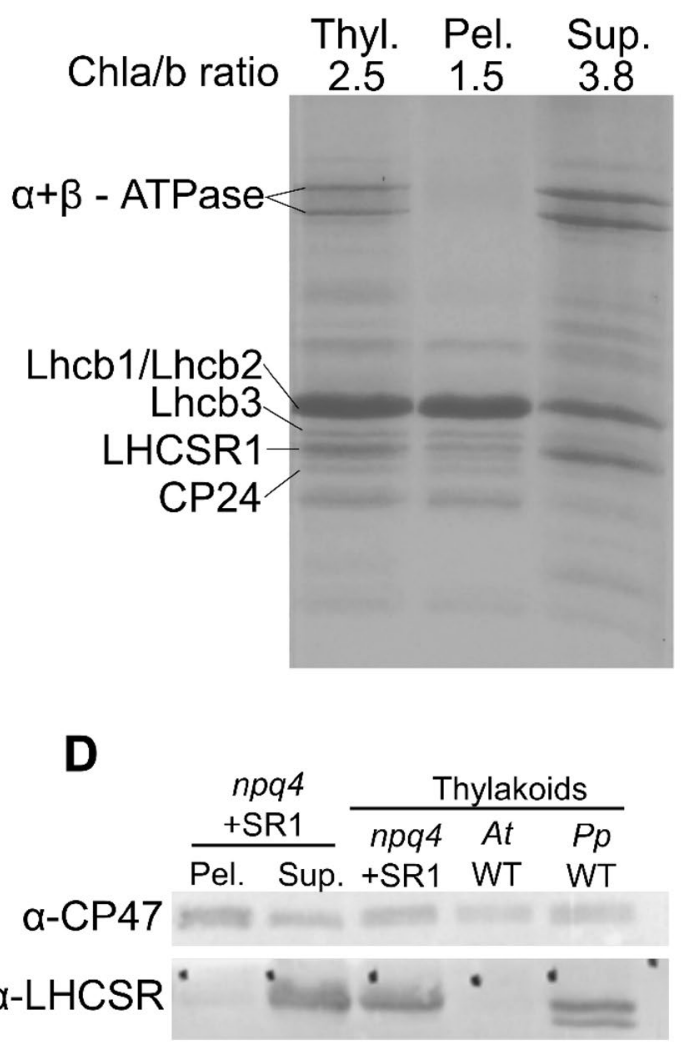

SDS-PAGE of fractionated thylakoid membranes from A. thaliana $n p q 4+$ LHCSR 1 using $0.47 \% \alpha$-DM. Thylakoid membranes (Thyl.), pellet enriched in grana fractions (Pel.) and the supernatant enriched in stroma membranes (Sup.). Gels were loaded on Chl basis, $4 \mu \mathrm{g}$ for thylakoids and $2.7 \mu \mathrm{g}$ for both the pellet and supernatant fractions. The $\mathrm{Chl} \mathrm{a} / \mathrm{b}$ ratio is indicated above the gel. $\mathbf{d}$ western blot analysis of the fractionated thylakoid membranes and thylakoids from $A$. thaliana $n p q 4, n p q 4+\mathrm{LHCSR} 1$, WT and P. patens $\mathrm{WT}$ as controls properties of recombinant LHCSR proteins either refolded in vitro or expressed in tobacco (Bonente et al. 2011; Pinnola et al. 2015b).

\section{NPQ activity of LHCSR1 in A. thaliana}

As previously mentioned, the NPQ activity of PSBS and LHCSR is additive and independent in $P$. patens plants (Alboresi et al. 2010; Gerotto et al. 2012). It was tested whether LHCSR1 could confer a light dependent in vivo 


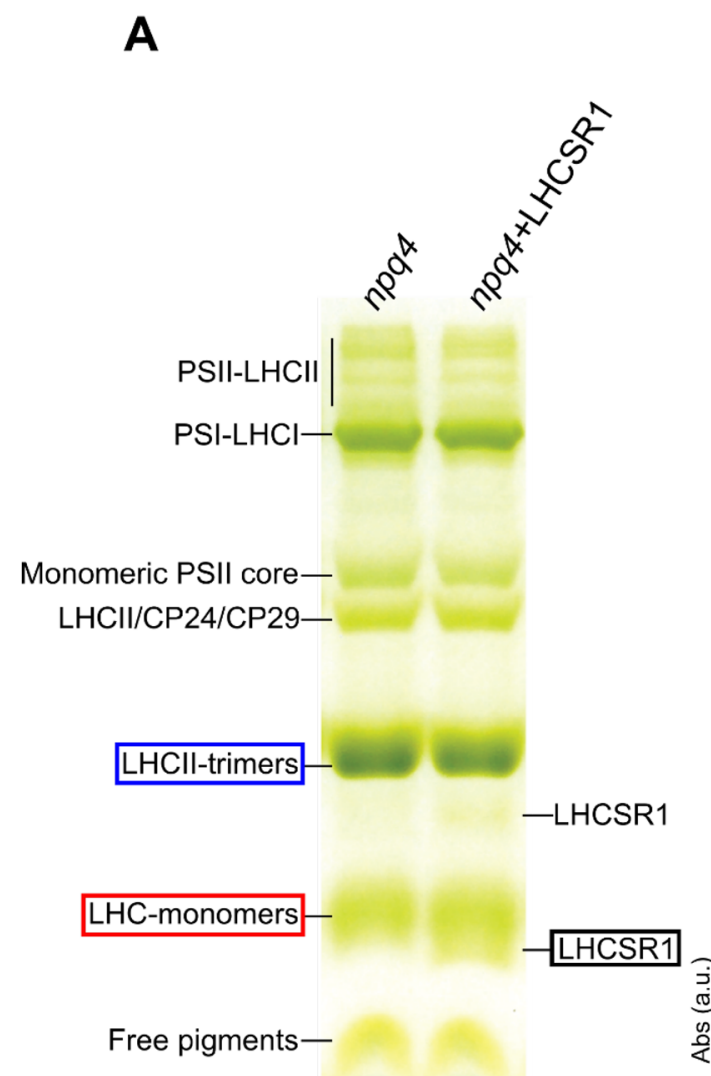

B

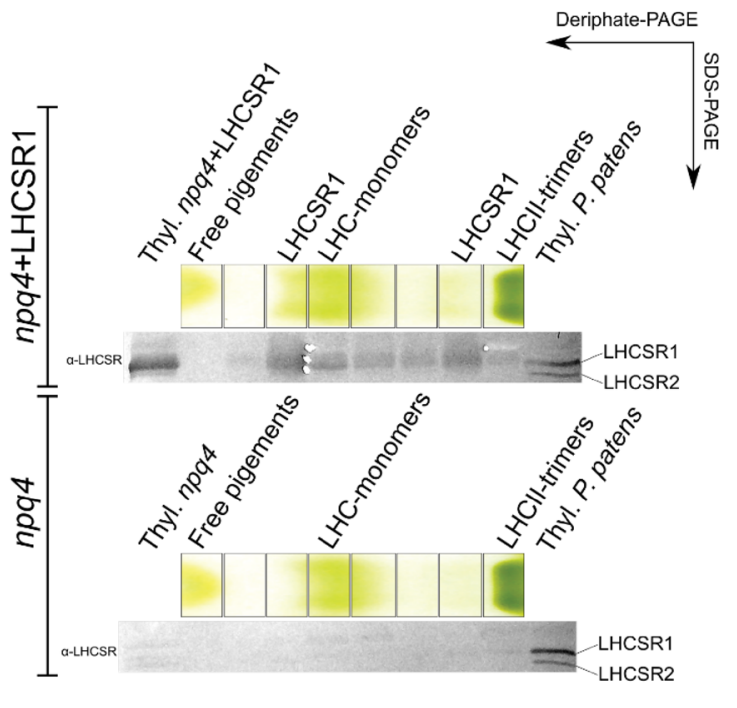

C

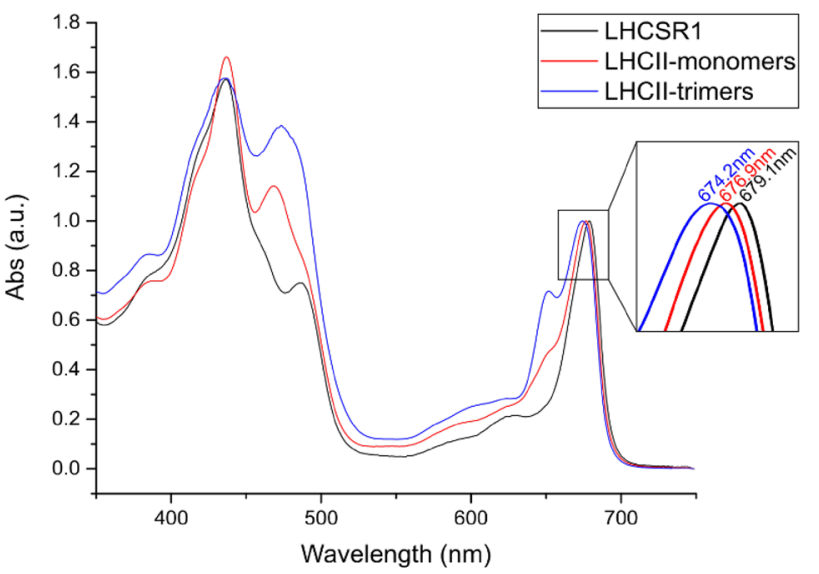

Fig. 2 Deriphat-PAGE analysis of $A$. thaliana thylakoid membrane protein complexes. a Thylakoid membranes $(30 \mu \mathrm{g}$ of Chl) of $n p q 4$ and $n p q 4+$ LHCSR 1 plants solubilized with $0.8 \%$ (w/v) $\alpha$-DM were subjected to Deriphat-PAGE. PSII and PSI complexes, together with various LHCs are indicated on the left side of the gel. Complexes more abundant in $n p q 4+$ LHCSR 1 than in $n p q 4$ plants are labeled as LHCSR1 on the right side of the gel. Colored rectangles correspond to the bands used for the absorption spectra analysis in (panel

quenching activity in the $A$. thaliana $n p q 4$ mutant. Therefore, the Chl fluorescence quenching of A. thaliana WT, $n p q 4$ plants and the complemented lines were measured using Chl fluorescence imaging. The protocol consisted of a 45 min dark adaptation of the leaves, followed by $5 \mathrm{~min}$ white actinic light $\left(1200 \mu \mathrm{mol}\right.$ photons $\left.\mathrm{m}^{-2} \mathrm{~s}^{-1}\right)$ and $5 \mathrm{~min}$ of dark recovery (Fig. 3). When the protocol was applied to dark-adapted leaves, only a small difference in quenching activity was observed, suggesting the expression of LHCSR1 did not confer significant NPQ activity (Fig. 3a). However, when the same protocol was applied for the second-time, larger differences between $n p q 4$ and $n p q 4+$ LHCSR 1 were observed (Fig. 3b). Two additional cycles of NPQ induction

c). b Deriphat-PAGE (7\%) of unstacked thylakoids from A. thaliana WT and $n p q 4+$ LHCSR1, solubilized in $0.8 \% \alpha$-DM. Bands were eluted in $10 \mathrm{mM} \mathrm{HEPES} / 0.03 \% \alpha$-DM. Eluted fractions were loaded on SDS-PAGE and immunoblotted against $\alpha$-LHCSR. c Absorption spectra of the bands taken from the Deriphat-PAGE (panel a), LHCSR1, LHCII-monomers and LHCII-trimers of A. thaliana $n p q 4+$ LHCSR 1

and relaxation were applied, where the differences were even further pronounced between the second and third measurement, the NPQ was very similar between the third and the fourth measurement (Fig. 3c, d). Interesting to note is that the $n p q 4$ mutant showed a characteristic transient increase of quenching at the first point in the dark after switching off the actinic light, this jump was not detected in plants containing PSBS nor was it detected in lines expressing LHCSR1 (Fig. 3a-d). Since the accuracy of these NPQ measurements depends on that of the Fm measurements. All the Fv/Fm values of the different NPQ measurements were included in Tables $\mathrm{S} 1$ and $\mathrm{S} 2 . F_{\mathrm{v}} / F_{\mathrm{m}}$ values were found to be very 
A

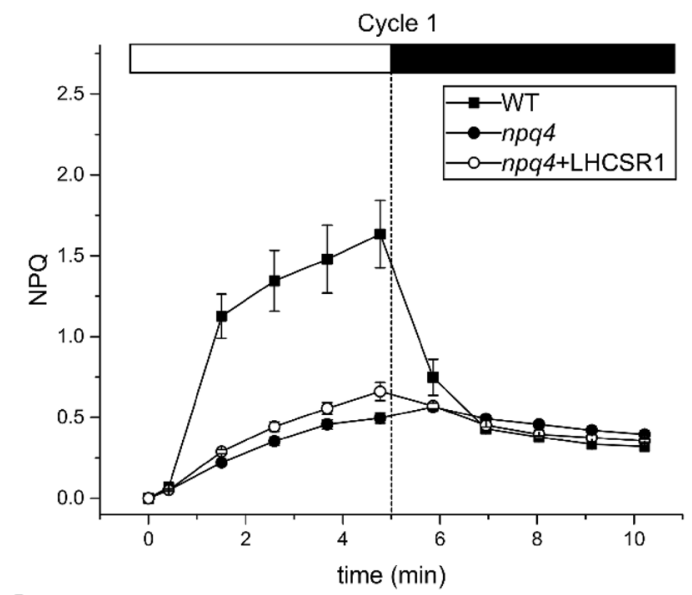

C

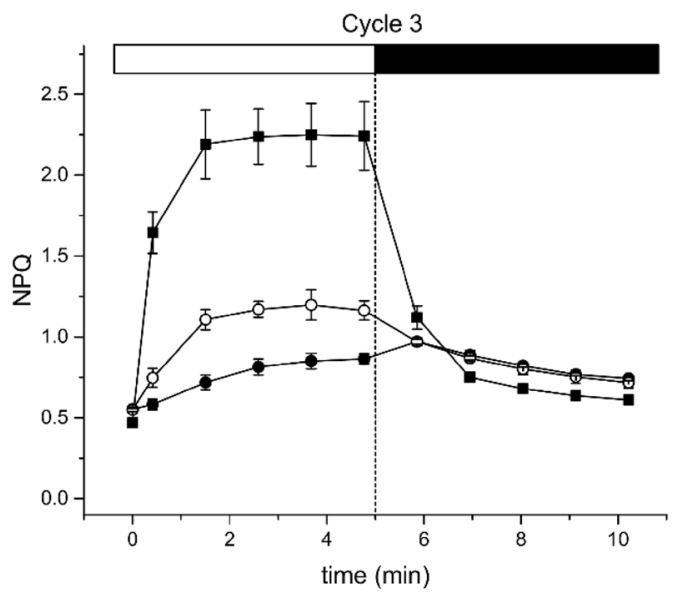

B

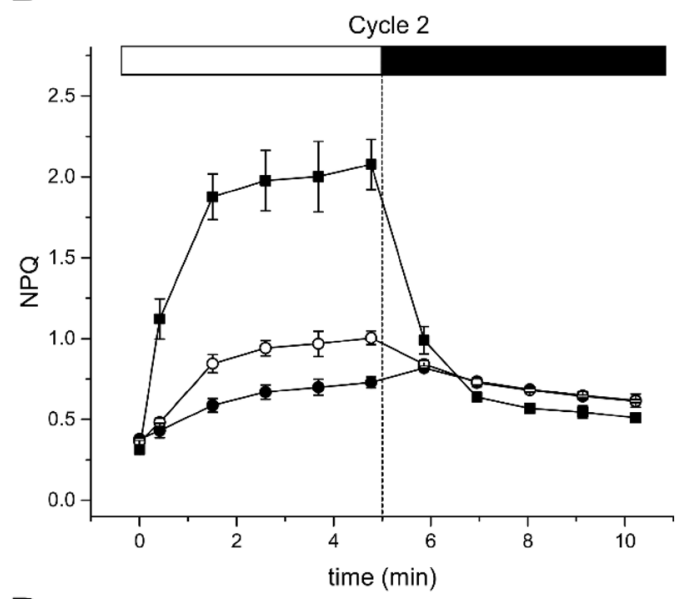

D

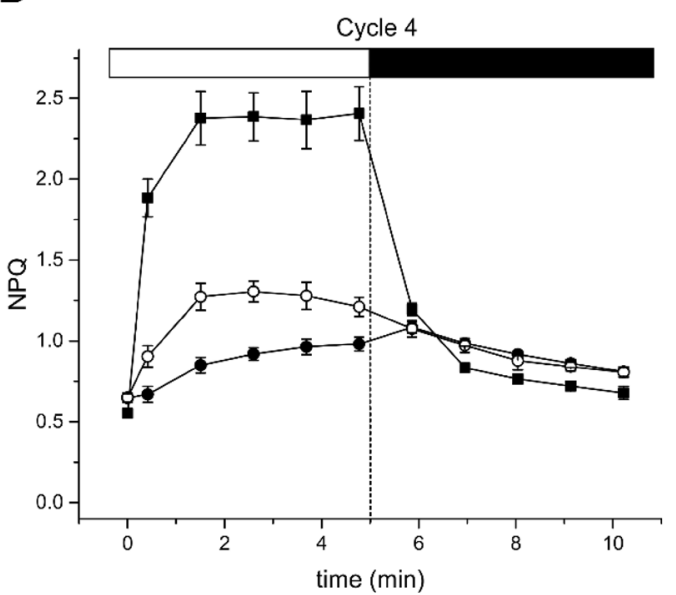

Fig. 3 NPQ kinetics $(n=4)$ in A. thaliana WT, $n p q 4$ and $n p q 4+$ LHCSR1. WT (Black squares), $n p q 4$ (black circles) and $n p q 4$ + LHCSR1 (open circles). Plants were dark adapted for $45 \mathrm{~min}$

similar for all the different LHCSR1 expressing lines (i.e. below $2 \%$ ).

\section{LHCSR1 and zeaxanthin synthesis}

Since Zea has a major influence on the quenching activity of LHCSR1 (Pinnola et al. 2013), the slow onset of LHCSR1dependent NPQ activity in A. thaliana suggests that Zea accumulation might be limiting. Therefore, the leaf pigment content was determined by HPLC analysis of the $n p q 4$ and two independent $n p q 4+$ LHCSR 1 lines during two cycles of $10 \mathrm{~min}$ illumination followed by a $10 \mathrm{~min}$ dark relaxation. Zea accumulated to the same level in both genotypes (Table S3) at the end of each dark or light phase while the 10-min dark periods did not allow for a decrease in Zea level. We conclude that the repeated cycles of illumination (Fig. 3) lead to an increased NPQ activity, which is consistent with the accumulation of Zea (Table S3). before the measurement, 4 cycles of 5 min actinic light $(1200 \mu \mathrm{mol}$ photons $\mathrm{m}^{-2} \mathrm{~s}^{-1}$ ) and $5 \mathrm{~min}$ dark, as described in the M\&M. The four cycles are depicted by $\mathbf{a}-\mathbf{d}$, respectively

\section{Correlation between LHCSR1 accumulation level and NPQ activity}

To verify whether NPQ activity correlated with the amount of LHCSR1, nine lines with different levels of NPQ were selected (Fig. 4a). Total leaf extracts were titrated with an $\alpha$-LHCSR polyclonal antibody (Fig. 4b). Both qE and total NPQ activities linearly correlated with the level of LHCSR1. An estimation of the $\mathrm{qE}$ was determined by differences between NPQ values recorded at the end of the 5-min light period and upon 2 min of dark relaxation, allowing for a rapid estimation of qE activity (Dall'Osto et al. 2014). The NPQ activity per LHCSR1 unit was lower in A. thaliana with respect to $P$. patens since LHCSR1-only mosses did show an NPQ score threefold higher than the complemented A. thaliana npq4 lines (Fig. 5a), while the level of LHCSR1 in P. patens psbs-lhcsr2 ko was only 1.2-fold higher (Fig. $\mathrm{S} 2)$. 
A

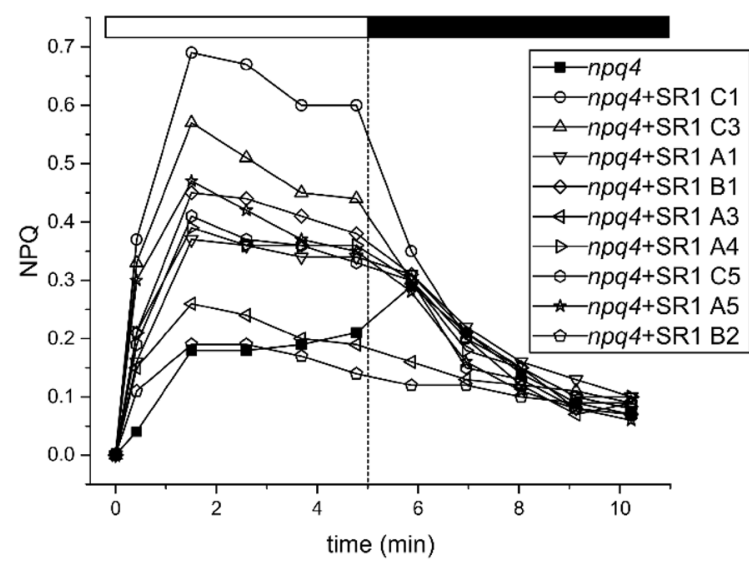

C

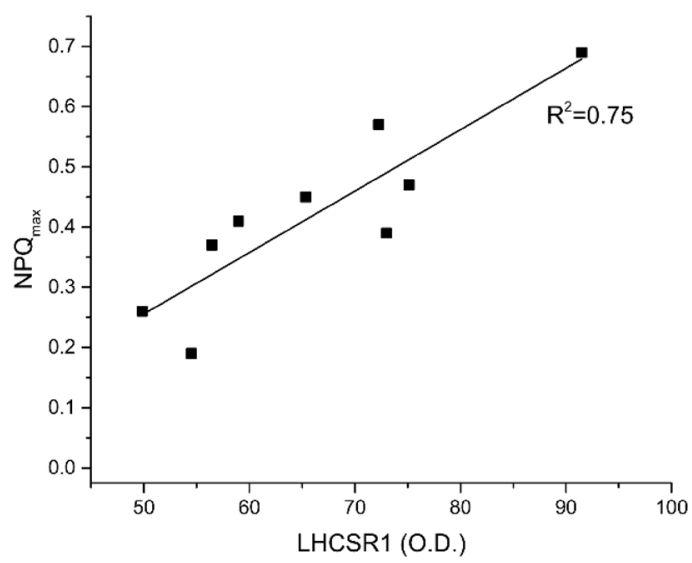

Fig. 4 Correlation between NPQ activity and LHCSR1 accumulation. NPQ measurements of the T2 generation of the $n p q 4+$ LHCSR 1 lines $(n=9)$. a. Leaves were dark adapted for $45 \mathrm{~min}$, pre-treated with $1200 \mu \mathrm{mol}$ photons $\mathrm{m}^{-2} \mathrm{~s}^{-1}$ of actinic light for $15 \mathrm{~min}$ and left to relax in the dark for 10 min before the NPQ measurement $\mathbf{b}$ After the NPQ measurement total leaf extracts from each line were loaded on an SDS-PAGE on a basis of $0.75 \mu \mathrm{g} \mathrm{Chl}$ and immuno-blotted

\section{LHCSR1-dependent NPQ in A. thaliana: dependence on light intensity}

In order to investigate the reasons for the low LHCSR1 activity in $A$. thaliana versus $P$. patens, we verified the hypothesis that mosses might differ from $A$. thaliana for their light intensity dependence of LHCSR 1 activity consistent with moss adaptation to shaded habitats. To this aim, three $n p q 4+$ LHCSR 1 lines with high and intermediate NPQ activity at $800 \mu \mathrm{mol}$ photons $\mathrm{m}^{-2} \mathrm{~s}^{-1}$ were selected and measured at a series of actinic light intensities: low light intensity $\left(100 \mu \mathrm{mol}\right.$ photons $\left.\mathrm{m}^{-2} \mathrm{~s}^{-1}\right)$ up to $1000 \mu \mathrm{mol}$ photons $\mathrm{m}^{-2} \mathrm{~s}^{-1}$. Before each measurement, leaves were dark-adapted for $45 \mathrm{~min}$, pre-treated with actinic light $\left(800 \mu \mathrm{mol}\right.$ photons $\left.\mathrm{m}^{-2} \mathrm{~s}^{-1}\right)$ for $15 \mathrm{~min}$ in order to accumulate equal Zea levels and left to relax
B
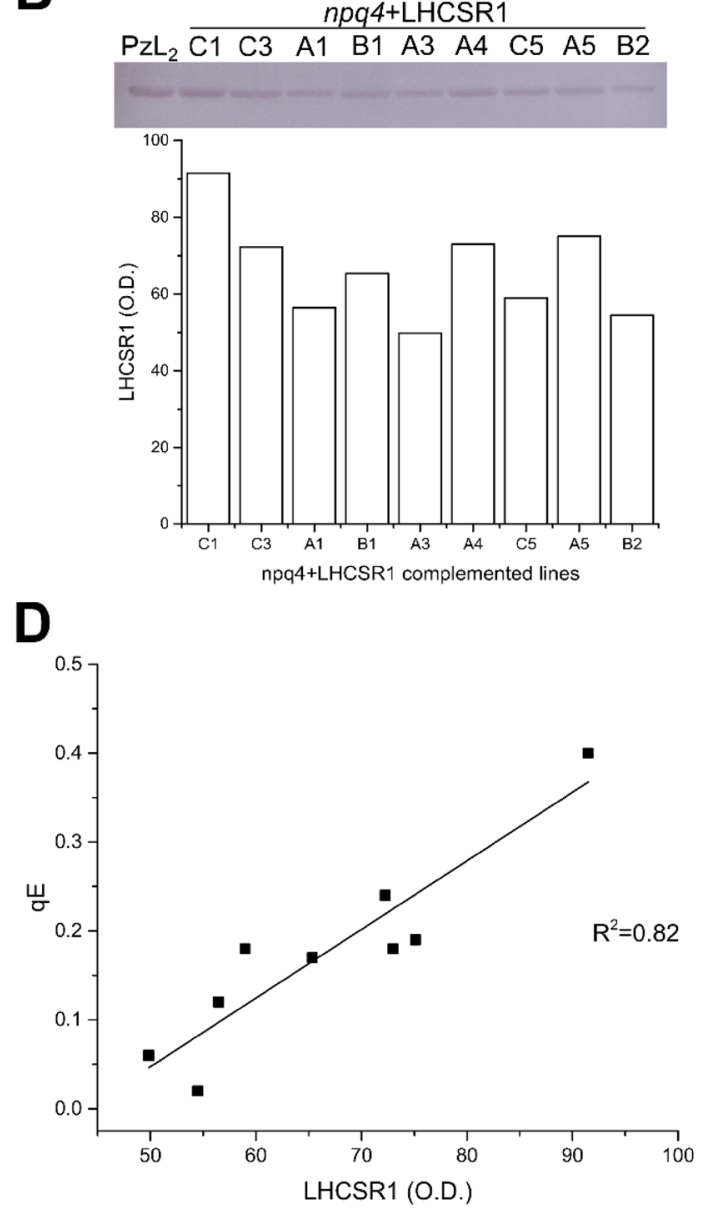

against $\alpha$-LHCSR antibodies. Thylakoids from P. patens psbs-lhcsr2 ko $\left(\mathrm{PzL}_{2}\right)$ were loaded as a control. The O.D. of LHCSR1 was determined. c Protein level plotted with the maximum NPQ, yielding a positive correlation of $R^{2}=0.75$. d Correlation between $\mathrm{qE}$ and the protein level $\left(R^{2}=0.82\right)$. $\mathrm{qE}$ recovery is calculated as the NPQ of the last point in the light phase minus the second point in the dark phase

for $10 \mathrm{~min}$ in the dark. Leaves from A. thaliana WT and $n p q 4$ plants of the same age were used as controls. At the lowest light intensity, transient NPQ was observed in all $A$. thaliana genotypes, which rapidly dropped, likely due to activation of the ATPase dissipating the $\Delta \mathrm{pH}$ for ATP synthesis (Fig. 6). However, as the intensity of actinic light increased, plants activated NPQ and the LHCSR1 complemented lines already showed activity at $200 \mu \mathrm{mol}$ photons $\mathrm{m}^{-2} \mathrm{~s}^{-1}$. Peak activity was reached after 2-3 min light exposure and the NPQ level was maximal at 400 and $600 \mu \mathrm{mol}$ photons $\mathrm{m}^{-2} \mathrm{~s}^{-1}$, with lower values at both lower and higher actinic light intensities. WT A. thaliana, besides showing at least twofold higher NPQ values, also did show strikingly different NPQ kinetics, monotonously rising under actinic light conditions and only relaxing when light was switched off. The partial relaxation of NPQ 
A

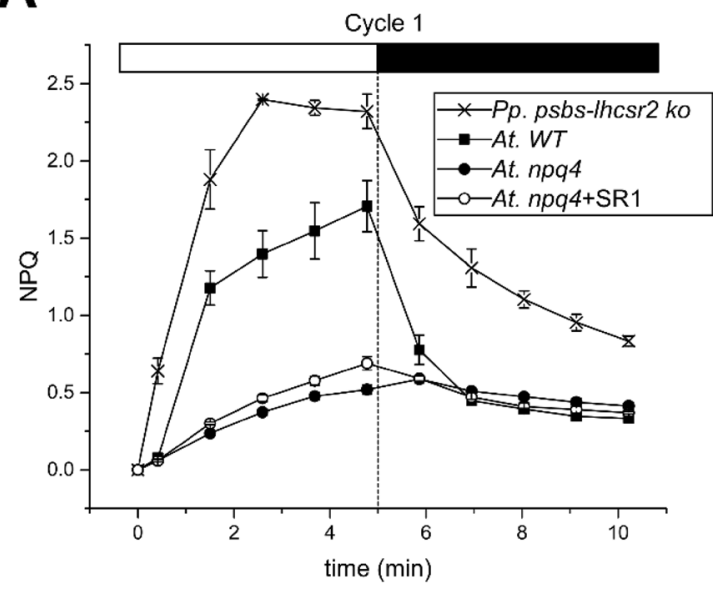

Fig. 5 Comparison of $\Delta \mathrm{pH}$ and NPQ between $P$. patens psbslhcsr 2 ko and A. thaliana. a Comparison of NPQ $(n=4)$ between A. thaliana WT, npq4, npq4+LHCSR1 (npq4+SR1) and P. patens psbs-lhcsr 2 ko. Fourth cycle of NPQ measurements at $1200 \mu \mathrm{mol}$

under actinic light could be explained by a relaxation of lumen acidity after $3 \mathrm{~min}$ of light treatment.

To verify whether the activation of LHCSR 1 might be affected by the amplitude of the $\mathrm{pH}$ gradient formation, we proceeded to measure the $\Delta \mathrm{pH}$ through thylakoid membranes at different light intensities. To this aim, chloroplasts of A. thaliana npq4 + LHCSR1 plants and P. patens psbs$l h c s r 2 \mathrm{ko}$ were illuminated in the presence of the fluorophore 9-aminoacridine (9-AA). 9-AA fluorescence is quenched upon protonation when the chemical diffuses through the thylakoids into the lumen dependent on the trans-membrane $\mathrm{pH}$ gradient. Figure $5 \mathrm{~b}$ shows the 9-AA fluorescence quenching in $A$. thaliana versus $P$. patens at different light intensities, implying a different capacity of building up a trans-thylakoid $\mathrm{pH}$ gradient between the two organisms. Despite the fact that $A$. thaliana was able to reach higher $\Delta \mathrm{pH}$ levels than P. patens, the NPQ activity of LHCSR1 in A. thaliana was lower with respect to P. patens (Fig. 5a).

\section{LHCSR1 expression and NPQ activity in xanthophyll biosynthesis mutants}

The above results imply that LHCSR 1 proteins expressed in A. thaliana $n p q 4$ can partially complement the lack of PSBS. When purified from Physcomitrella patens, LHCSR1 binds lutein (Lut) and Vio, part of which are substituted by Zea upon high light treatment (Pinnola et al. 2013). To identify the role of these xanthophylls for activation of LHCSR 1 in A. thaliana we proceeded with the transformation of the lhcsrl-gene in the following A. thaliana double mutants: (i) npqlnpq4, unable to accumulate Zea due to the lack of violaxanthin de-epoxidase ( $v d e$ ); (ii) $n p q 2 n p q 4$, a mutant
B

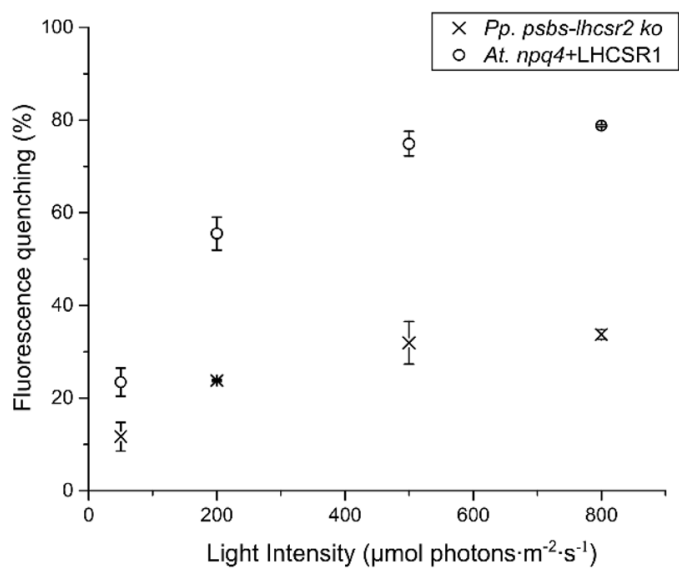

photons $\mathrm{m}^{-2} \mathrm{~s}^{-1}$ (5 min light and 5 min dark relaxation). b 9-aminoacridin measurements $(n=3)$ in isolated chloroplasts of A. thaliana $n p q 4+$ LHCSR 1 and $P$. patens psbs-lhcsr 2 ko at different light intensities $\left(50,200,500\right.$ and $800 \mu \mathrm{mol}$ photons $\mathrm{m}^{-2} \mathrm{~s}^{-1}$ of red light)

accumulating Zea due to the absence of zeaxanthin epoxidase (zep) (iii) lut2npq4, the lutein-less genotype defective in the lycopene $\varepsilon$-cyclase activity, which compensates missing Lut with increased levels of Vio.

Transformation of the npq1npq4 mutant with LHCSR1 and selection in hygromycin yielded 16 stable lines, which accumulated LHCSR1 as assessed by western blot analysis (Fig. S5b), implying LHCSR 1 can be expressed and accumulated in the absence of Zea (Fig. S5b). No major differences in the size or shape of the transformed plants were detected (Fig. S5a). The NPQ activity of npqlnpq4 plants and three independent complemented lines was measured by video-imaging following the initial protocol (see M\&M). The quenching activity of the complemented lines was the same as in the npqlnpq4 background and did not increase during the subsequent cycles of illumination, failing to reveal any difference between $n p q 1 n p q 4$ (i.e. control) and the complemented npqInpq4+LHCSR1 plants (Fig. 7a, b; Fig. S6a-d). This result is in agreement with previous reports in the homologous system $P$. patens showing that LHCSR1 requires Zea for quenching (Pinnola et al. 2013).

The $n p q 2$ mutant lacks Vio and accumulates full levels of Zea as well as Lut (Niyogi et al. 1998; Peers et al. 2009). Complementation of the $n p q 2 n p q 4$ mutant yielded three plants accumulating LHCSR1 (Fig. S7). NPQ activity differed with respect to $n p q 4+$ LHCSR 1 in that it appeared already during the first cycle of illumination in dark-adapted plants (Fig. 7c, d; Fig. S8a-d). Furthermore, the total amount of NPQ in these plants was much higher, reaching up to $70 \%$ of $A$. thaliana WT. These observations indicate that the build-up and level of Zea are one of the limiting factors in the NPQ activity of LHCSR1. During the fourth 
A

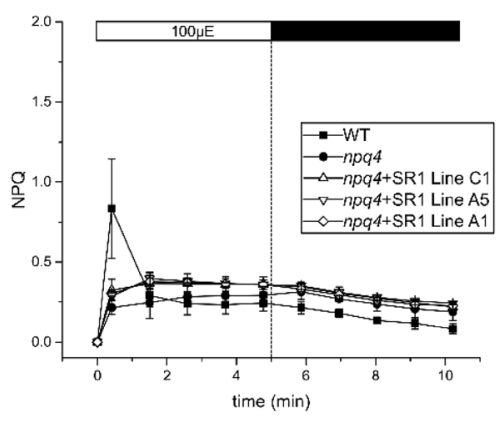

D

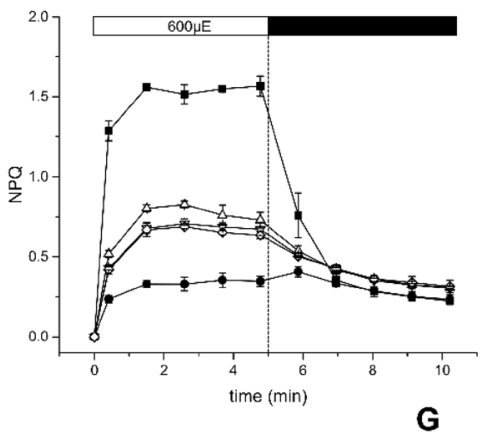

B

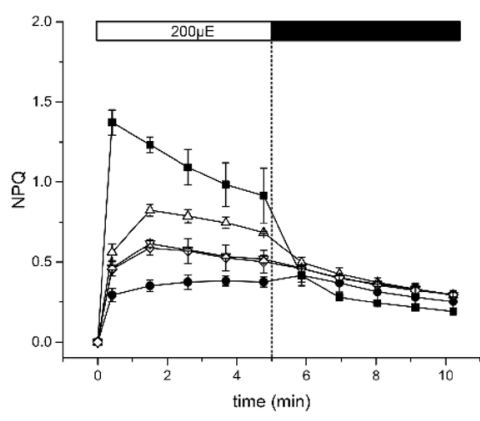

E

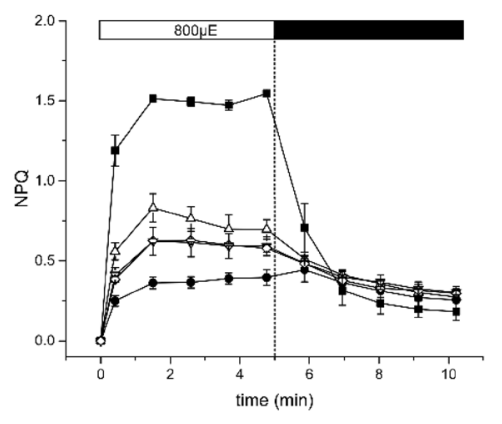

C

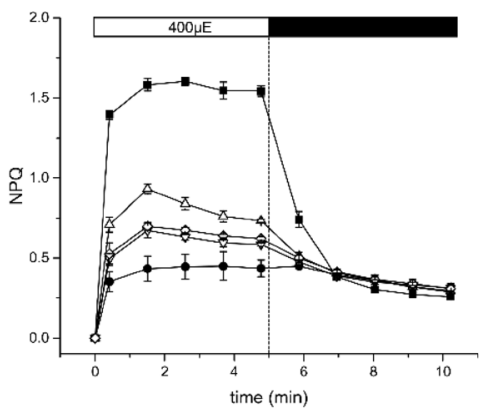

$\mathbf{F}$

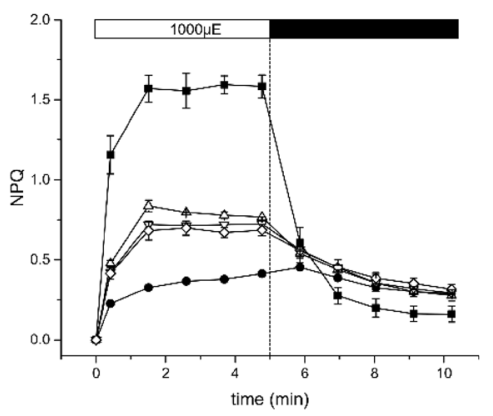

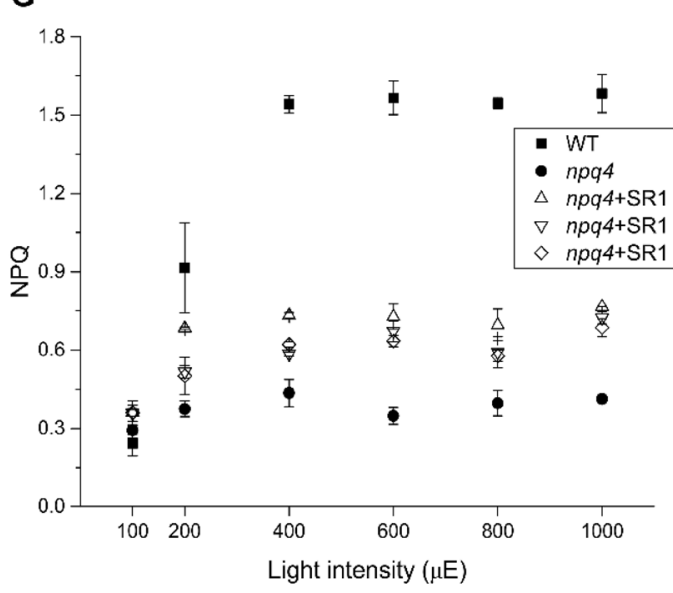

Fig. 6 NPQ activity of $n p q 4+$ LHCSR 1 lines in various light intensities. Three different $A$. thaliana npq4 +LHCSR1 lines with high and intermediate NPQ activation (line $\mathrm{C} 1, \mathrm{~A} 1$ and A5) were tested in a variety of actinic light intensities. Leaves $(n=3)$ were dark adapted for $45 \mathrm{~min}$, pre-treated with $800 \mu \mathrm{mol}$ photons $\mathrm{m}^{-2} \mathrm{~s}^{-1}$ of actinic light for $15 \mathrm{~min}$ and left to relax in the dark for $10 \mathrm{~min}$ before the NPQ measurement. Each measurement corresponds to one single NPQ

cycle the maximal NPQ was reached after 2 min and showed the same partial relaxation kinetics as the $n p q 4+$ LHCSR 1 . This relaxation, however, could already be observed during the third cycle in the $n p q 2 n p q 4$ complemented lines, but not in $n p q 4$ + LHCSR1.

LHCSR 1 expression in the lut2npq4 yielded no major phenotypic differences between control and LHCSR1-complemented lut2npq4 plants (Fig. S9a). Total leaf extracts from the complemented lines were analyzed by western cycle of 5 min different with different actinic light intensities and 5 min dark recovery. The actinic light intensities used were: 100, 200, 400, 600, 800 and $1000 \mu$ mol photons $\mathrm{m}^{-2} \mathrm{~s}^{-1}(\mu \mathrm{E})$ from $\mathbf{a}-\mathbf{f}$, respectively. Leaves from A. thaliana WT and $n p q 4$ were used as controls. g NPQ of the last point in the light plotted against the different light intensities

blot with the $\alpha$-LHCSR antibodies (Fig. S9b), showing that the protein was processed to its mature form without Lut. Using the 4-cycle actinic light protocol, the control and complemented lines were essentially indistinguishable in the first cycle (Fig. 7e). However, upon the second cycle the curves of the different genotypes became more shifted towards higher values with the exception of lut $2 n p q 4$, which remained unchanged (Fig. S10). Two additional cycles further increased the difference in NPQ between lut2npq4 and 
A

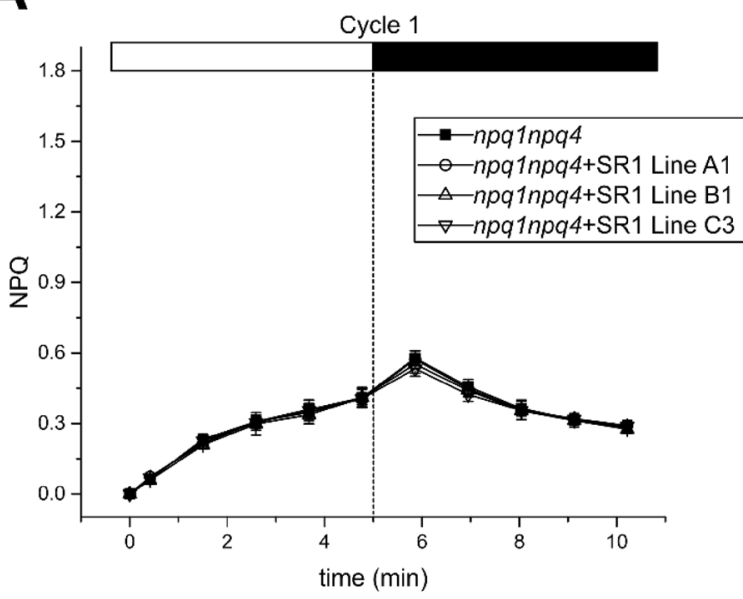

C

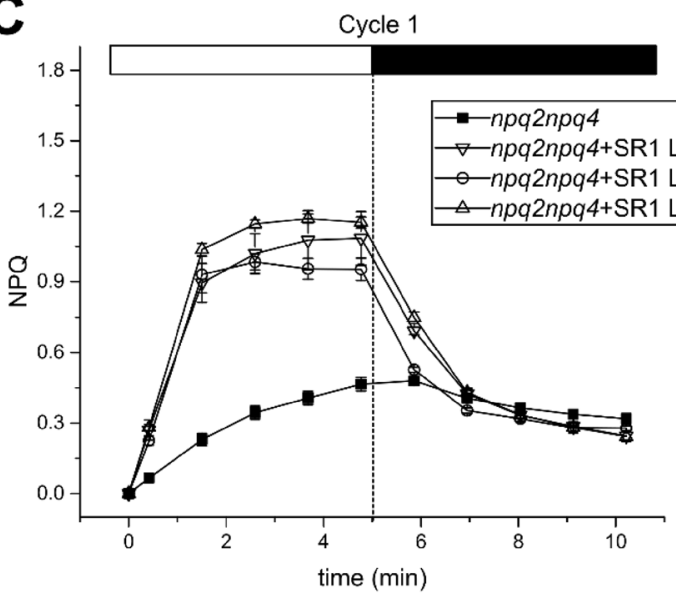

E

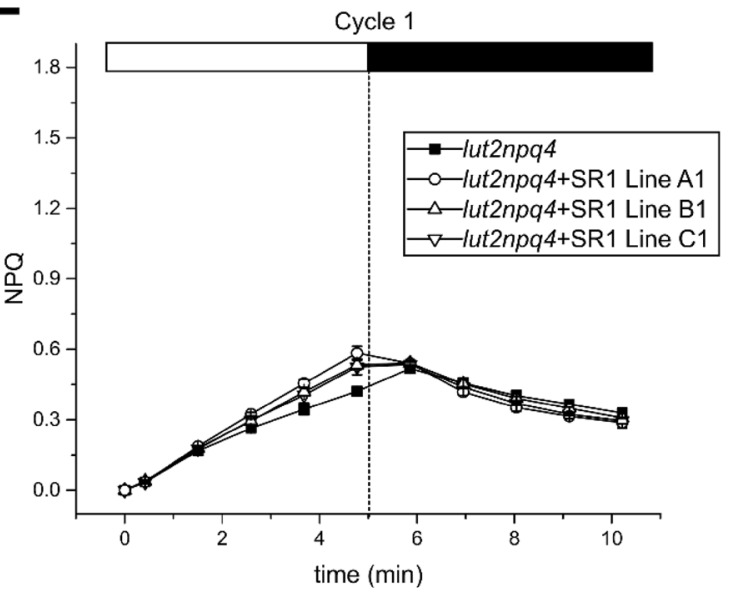

B

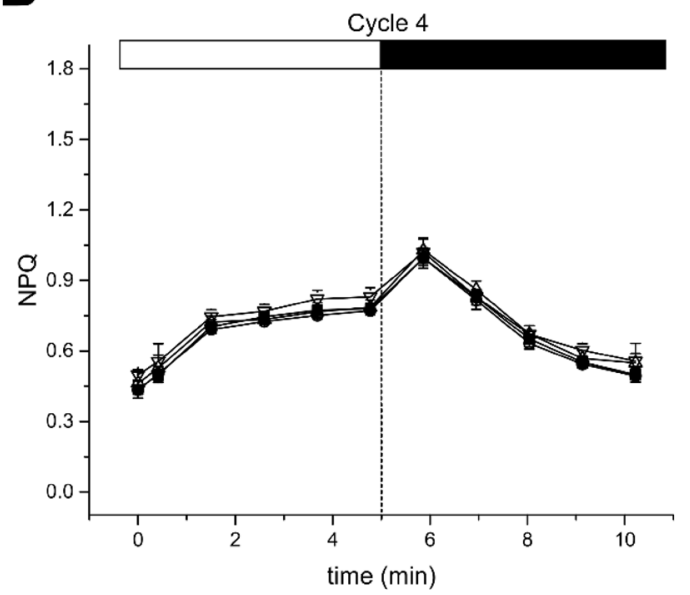

D

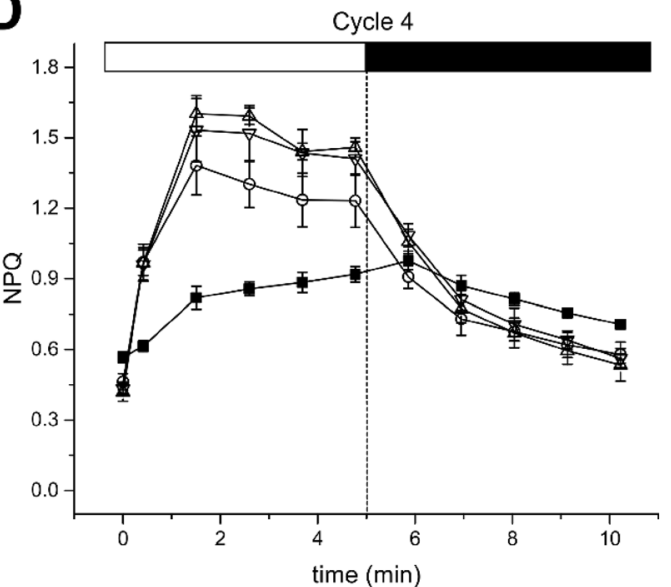

$\mathbf{F}$

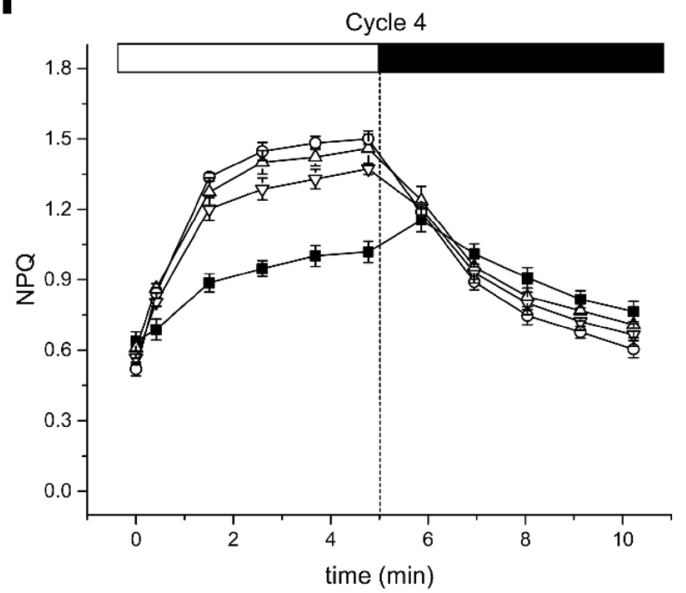

Fig. 7 NPQ measurements in A. thaliana mutants, lacking specific xanthophyll's, complemented with LHCSR1. Four successive NPQ cycles were measured $(n=3)$ as described in M\&M. The first and fourth cycle are shown for each complemented mutant. a, b first and fourth NPQ measurement in npqlnp4 and three independent npq1npq4 lines complemented with LHCSR1 (npq1npq4+SR1). c, d first and fourth NPQ measurement in $n p q 2 n p q 4$ and three $n p q 2 n p q 4$ lines complemented with LHCSR1 (npq2npq4+SR1). e, f first and fourth NPQ measurement in lut2npq4 and three lut2npq4 lines complemented with LHCSR1 (lut2npq4 +SR1) 
the complemented lines (Fig. 7f). Two features characterized these measurements with respect to the $n p q 4+$ LHCSR 1 genotype: first, the higher level of $\mathrm{qE}$ obtained in the lut$2 n p q 4$ + LHCSR1 with respect to $n p q 4+$ LHCSR1; second, that the maximal NPQ values were obtained at the end of the illumination ( $5 \mathrm{~min}$ ) rather than at the second minute as previously observed with the $n p q 4+$ LHCSR 1 and $n p q 2 n p q 4$ + LHCSR 1 genotypes, consistent with a delay in reaching full de-epoxidation of Vio bound to slowly exchanging binding sites (Morosinotto et al. 2002).

\section{Discussion}

While expression in tobacco has proven instrumental for purification of the LHCSR1 protein for biochemical, spectroscopic and structural studies (Pinnola et al. 2015b, 2016, 2017; Kondo et al. 2017, 2019), A. thaliana is a choice for functional studies due to the availability of a large collection of mutants and the easiness of transformation procedures. Here, we show that the moss LHCSR 1 protein can be expressed in A. thaliana to form a pigment-protein complex indistinguishable from the holoprotein purified from $P$. patens as judged from the mass spectrometric analysis and visible absorption spectra (Fig. 2c, S3). Also consistent with previous reports, LHCSR1 was found both as a monomer and dimer in non-denaturing green gels, suggesting its aggregation state in thylakoids is a dimer which, in part, monomerizes during solubilization and fractionation (Pinnola et al. 2015b). LHCSR1 purified from P. patens, binds Chl $a$, Lut and Vio with sub-stoichiometric levels of Chl $b$, where Vio is largely substituted by Zea during high light treatment (Pinnola et al. 2013, 2015b). Here, we show that LHCSR1 from $P$. patens can be expressed in A. thaliana npq4 mutants at levels comparable to that found in moss. Due to the absence of PSBS, the host lines did not show NPQ activity upon high light treatment (Li et al. 2000). Thus NPQ detected in complemented plants can be attributed to LHCSR 1 based on the following observations: (i) quenching was only observed in LHCSR-complemented genotypes (Fig. 3); (ii) quenching was proportional to the level of LHCSR1 accumulation (Fig. 4); (iii) no LHCSR1dependent quenching activity was observed in the npqlnpq4 background, lacking Zea (Fig. 7a, b), in agreement with the observation that the vde ko mutant in P. patens lost $95 \%$ of quenching activity (Pinnola et al. 2013); (iv) higher and fast-developing quenching was observed upon expression in the npq2npq4 background lacking Vio and constitutively accumulating Zea (Fig. 7c, d). All these features closely reproduce the properties of LHCSR 1 activity in the moss, implying the observed NPQ could be bona fide attributed to LHCSR1. LHCSR1 was correctly addressed to the thylakoid membranes with an apparent molecular weight identical to the LHCSR1 of $P$. patens, as observed in SDS-PAGE gels (Fig. 1), implying a correct targeting and processing of the pre-protein encoded by the construct. We then proceeded to identify the factors which determine the level of NPQ activity, including the accumulation in the thylakoids, the availability of the Zea co-factor and the co-localization with PSII whose fluorescence is quenched during NPQ. The level of LHCSR1 in A. thaliana npq4 was slightly lower $(82.8 \pm 1.8 \%)$ with respect to the P. patens psbs-lhcsr 2 ko. Yet, while LHCSR1 is the major contributor to NPQ activity in moss (Alboresi et al. 2010) providing an NPQ activity of 3.2, the NPQ activity in A. thaliana was lower. Besides the lower levels of Zea found in A. thaliana, this can be explained by (i) a lower level of LHCSR1 activation by a difference in lumen acidification; (ii) a different localization in thylakoids with respect to the PSII antenna system, which is the major fluorescence emitter in vivo; or (iii) the lack of one or more interaction partner(s) acting as a docking site for connecting the quenching site within the LHCSR1 to the PSII antenna system. To test the first hypothesis, we proceeded to determine the $\Delta \mathrm{pH}$ in $n p q 4+$ LHCSR 1 and $P$. patens psbs-lhcsr 2 ko chloroplasts by the 9-AA quenching method and showed that $\Delta \mathrm{pH}$ formation is higher in A. thaliana with respect to $P$. patens. This would suggest that the lower NPQ in A. thaliana might be due to over-acidification of the lumen. LHCSR1, however, was not active at $100 \mu \mathrm{mol}$ photons $\mathrm{m}^{-2} \mathrm{~s}^{-1}$ in A. thaliana, a light intensity where the $\Delta \mathrm{pH}$ is comparable to that found in P. patens. The highest quenching activity of LHCSR1 in A. thaliana was found at 400 and $600 \mu \mathrm{mol}$ photons $\mathrm{m}^{-2} \mathrm{~s}^{-1}$, light intensities where the $\Delta \mathrm{pH}$ is already much higher in A. thaliana in comparison to $P$. patens. Making it unlikely that over-acidification is the reason for the lower quenching activity. Hypothesis (ii) appears to be relevant in determining a reduced NPQ since the membrane fractionation experiment located LHCSR 1 in the stroma membranes (Fig. 1c, d), consistent with previous findings in the moss. It should be noted that a large fraction of LHCII is located in moss stroma membranes (Pinnola et al. 2015b), while higher plants show extreme lateral heterogeneity with LHCII being located almost exclusively in the grana (Bassi et al. 1988; Pribil et al. 2014). Thus, interaction between LHCSR1 and PSII antennas appears to be restricted to grana margins, implying that only a low fraction of PSII supercomplexes might be involved. This is likely to decrease the quenching efficiency of $n p q 4+$ LHCSR 1 plants with respect to WT A. thaliana, with PSBS localized in grana partitions together with PSII antenna (Pinnola et al. 2015b). Hypothesis (iii) is synergic with (ii). In fact, work in A. thaliana (Pietrzykowska et al. 2014) and C. reinhardtii (Elrad et al. 2002; Ferrante et al. 2012) has shown that quenching requires specific members of the LHC protein family with which PSBS and LHCSR interact (Girolomoni et al. 2016). It is well possible that one or more LHCSR1-interacting 
proteins in moss are not conserved in A. thaliana and/or that the interaction is partially impaired.

In addition to differences in amplitude, the kinetics of LHCSR1-dependent quenching is also different in transgenic A. thaliana versus $P$. patens in some aspects: first, quenching is activated only upon pre-treatment with high light while in the moss it is evident at the first light exposure of dark-adapted mosses (Fig. S11). Explanation of this behavior is provided by the results of complemented $n p q 1 n p q 4$ and $n p q 2 n p q 4$ genotypes: plants from the former genotype showed no quenching activity due to the absence of Zea, despite LHCSR1 accumulation in the thylakoids. Plants from the latter genotype, the $n p q 2 n p q 4$ which are fully endowed with Zea, not only show a higher quenching activity, but also, a faster activation, i.e. at the first cycle of illumination (Fig. 7c) rather than at the third as in the $n p q 4$ plants expressing LHCSR1. Even though the npq2npq4 mutant contains 2.5 folds more Zea in comparison to high light adapted $P$. patens (Dall'Osto et al. 2005; Pinnola et al. 2013), the level of NPQ was lower than found in both $P$. patens LHCSR1-only or A. thaliana WT, implying that Zea was not the only limiting factor for the quenching activity of LHCSR1 in A. thaliana. Interestingly, the kinetics of the De-epoxidation Index (DEP) are very similar to the kinetics of quenching in both A. thaliana and P. patens: in A. thaliana the maximal DEP was reached after $10 \mathrm{~min}$ (Dall'Osto et al. 2017), in P. patens a DEP comparable to the maximum of A. thaliana was already reached after $1.5 \mathrm{~min}$ (Fig. S12). This suggests that there is simply not enough Zea in $A$. thaliana to completely activate LHCSR1, which is consistent with the enhanced activity of LHCSR1 in the npq2npq4 background where Zea availability was constitutive rather than induced by light exposure. The lut2npq 4 complemented lines, lacking Lut, showed activity in NPQ, meaning that Lut is not an absolute requirement for the in vivo quenching in LHCSR1. This is consistent with the recent finding that the major quenching mechanism in isolated LHCSR 1 is energy transfer from $\mathrm{Chl}$ to $\mathrm{S} 1$ state of Zea followed by rapid relaxation to ground state, while transient formation of Lut radical cation was low (Pinnola et al. 2016). In the lut2npq4 complemented lines the peak NPQ activity was observed at later times upon each onset of the actinic light. While the NPQ peak was observed after 2 min of light exposure in $n p q 4+$ LHCSR 1 , the NPQ of lut $2 n p q 4+$ LHCSR 1 steadily raised till the end of the light phase. This behavior can be explained based on the enhanced Vio content in the L2 binding site, due to compensation for the missing Lut (Pogson et al. 1996; Dall'Osto et al. 2006). Vio is an inhibitor of quenching reactions (Niyogi et al. 1998; Ruban et al. 1998) and replaces Lut in sites L1 and L2 of LHCII proteins (Croce et al. 1999; Dall'Osto et al. 2006). The kinetic difference can be explained with two independent events of xanthophyll exchange: one at site L1, which substitutes Vio for
Zea, while the second event is the exchange at site L2 which, are kinetically different in LHC proteins (Morosinotto et al. 2002). Since occupation of L1 site by Lut or Zea is essential for the NPQ activity (Dall'Osto et al. 2006), the onset of NPQ was slower in lut2npq4+LHCSR 1 with respect to $n p q 2 n p q 4$ + LHCSR1 or $n p q 4$ + LHCSR1 which have Lut in site L1 already in the dark and only need to perform the Vio to Zea exchange in site L2. Future research will need to devise new methods for assessing the xanthophyll composition of LHCSR1 in real time as well as other LHC proteins essential for quenching reactions in plants, mosses and algae since the exchange might be fast and reversible in minutes. It remains to be explained why the $n p q 2 n p q 4+$ LHCSR 1 or $n p q 4+$ LHCSR 1 show an NPQ kinetic rapidly climbing to a peak and then relaxing or remaining constant during the remaining light period. We suggest this depends on the $\Delta \mathrm{pH}+\Delta \psi$ gradient through the thylakoid membrane that appears to be different in $A$. thaliana versus $P$. patens. 9AA quenching showed a lower $\Delta \mathrm{pH}$ contribution in $P$. patens and yet LHCSR1 might respond to $\Delta \psi$ as well. Upon transition from dark to excess light, a transient lumen acidification is reached due to the contribution of cyclic electron flow, recycling excess reducing power into over-reduction of plastoquinol and additional proton transport (Munekage et al. 2004). The $\Delta \mathrm{pH}$ and/or $\Delta \psi$ past transient cyclic burst might be insufficient to sustain full LHCSR1 activation in A. thaliana.

\section{Conclusion}

We show that heterologous expression of LHCSR1 in $A$. thaliana npq4 mutant yields a pigment-binding protein with properties reproducing those of LHCSR1 from the homologous system $P$. patens. The protein is active in NPQ, yet the induction requires sustained light treatment due to the need for Zea build-up. Reasons for a decreased NPQ include (a) insufficient Zea accumulation in A. thaliana with respect to $P$. patens for full NPQ activity and (b) the localization of the protein in the stromal membranes of thylakoids which is rich in highly fluorescent LHCII in mosses but not in plants (Pinnola et al. 2015a). The level of quenching in $n p q 2 n p q 4+$ LHCSR1 (endowed with full Lut and Zea levels) recovered up to $70 \%$ with respect to A. thaliana WT, proving that LHCSR 1 can be highly functional in vascular plants. Furthermore, we prove that this system is sensitive to physiological differences which makes A. thaliana an excellent organism for the analysis of LHCSR activity. Indeed, we could assess that Lut was not an absolute requirement for in vivo quenching in LHCSR 1 , since quenching activity was obtained in lut2npq4 + LHCSR1 plants.

The primary target of plants is survival; thus, they favor thermal dissipation over fast growth. But an NPQ 
mechanism with low activity in moderate light intensity and full activation in extreme stress conditions only, might allow for optimizing both growth and stress resistance. This could be the case for the LHCSR 1 protein expressed in the heterologous systems which exhibits an activity, even if low in the WT and higher in the $n p q 2$ background, in stressing conditions. Future work will evaluate the growth performance (productivity) of these plants in different constant light condition as well as in fluctuating light which is the most stressing condition and mimics the natural environment.

Finally, since the NPQ activity of LHCSR 1 and PSBS is cumulative, suggesting they have different interaction partners, future work with deletion mutants of specific LHC proteins will pinpoint the interaction partners of LHCSR 1 and help us elucidate how PSBS and LHCSR1 evolved.

Acknowledgements The research was supported by the Marie Curie Actions Initial Training Networks SE2B (675006-SE2B) and ACCLIPHOT (316427-ACCLIPHOT).

\section{Compliance with ethical standards}

Conflict of interest The authors declare that they have no conflict of interest.

Open Access This article is distributed under the terms of the Creative Commons Attribution 4.0 International License (http://creativeco mmons.org/licenses/by/4.0/), which permits unrestricted use, distribution, and reproduction in any medium, provided you give appropriate credit to the original author(s) and the source, provide a link to the Creative Commons license, and indicate if changes were made.

\section{References}

Ahn TK, Avenson TJ, Ballottari M et al (2008) Architecture of a charge-transfer state regulating light harvesting in a plant antenna protein. Science 320:794-797. https://doi.org/10.1126/scien ce. 1154800

Alboresi A, Gerotto C, Giacometti GM et al (2010) Physcomitrella patens mutants affected on heat dissipation clarify the evolution of photoprotection mechanisms upon land colonization. Proc Natl Acad Sci USA 107:11128-11133. https://doi.org/10.1073/ pnas. 1002873107

Allorent G, Tokutsu R, Roach T et al (2013) A dual strategy to cope with high light in Chlamydomonas reinhardtii. Plant Cell 25:545557. https://doi.org/10.1105/tpc.112.108274

Ashton NW, Grimsley NH, Cove DJ (1979) Analysis of gametophytic development in the moss, Physcomitrella patens, using auxin and cytokinin resistant mutants. Planta 144:427-435. https://doi. org/10.1007/BF00380118

Baker NR (2008) Chlorophyll fluorescence: a probe of photosynthesis in vivo. Annu Rev Plant Biol 59:89-113. https://doi.org/10.1146/ annurev.arplant.59.032607.092759

Ballottari M, Truong TB, De Re E et al (2016) Identification of pHsensing sites in the light harvesting complex stress-related 3 protein essential for triggering non-photochemical quenching in Chlamydomonas reinhardtii. J Biol Chem 291:7334-7346. https ://doi.org/10.1074/jbc.M115.704601
Bassi R, Giacometti GM, Simpson DJ (1988) Changes in the organization of stroma membranes induced by in vivo state 1-state 2 transition. Biochim Biophys Acta Bioenerg 935:152-165. https ://doi.org/10.1016/0005-2728(88)90212-5

Berthold DA, Babcock GT, Yocum CF (1981) A highly resolved, oxygen-evolving photosystem II preparation from spinach thylakoid membranes. FEBS Lett 134:231-234. https://doi. org/10.1016/0014-5793(81)80608-4

Bonente G, Ballottari M, Truong TB et al (2011) Analysis of LhcSR3, a protein essential for feedback de-excitation in the green alga Chlamydomonas reinhardtii. PLoS Biol 9:e1000577. https://doi. org/10.1371/journal.pbio.1000577

Brooks MD, Sylak-Glassman EJ, Fleming GR, Niyogi KK (2013) A thioredoxin-like/-propeller protein maintains the efficiency of light harvesting in Arabidopsis. Proc Natl Acad Sci USA 110:E2733E2740. https://doi.org/10.1073/pnas.1305443110

Clough SJ, Bent AF (1998) Floral dip: a simplified method for Agrobacterium-mediated transformation of Arabidopsis thaliana. Plant J 16:735-743. https://doi.org/10.1046/j.1365-313x.1998.00343.x

Croce R, Weiss S, Bassi R (1999) Carotenoid-binding sites of the major light-harvesting complex II of higher plants. J Biol Chem 274:29613-29623. https://doi.org/10.1074/JBC.274.42.29613

Dall'Osto L, Caffarri S, Bassi R (2005) A mechanism of nonphotochemical energy dissipation, independent from PsbS, revealed by a conformational change in the antenna protein CP26. Plant Cell 17:1217-1232. https://doi.org/10.1105/tpc.104.030601

Dall'Osto L, Lico C, Alric J et al (2006) Lutein is needed for efficient chlorophyll triplet quenching in the major LHCII antenna complex of higher plants and effective photoprotection in vivo under strong light. BMC Plant Biol 6:32. https://doi. org/10.1186/1471-2229-6-32

Dall'Osto L, Cazzaniga S, Wada M, Bassi R (2014) On the origin of a slowly reversible fluorescence decay component in the Arabidopsis npq4 mutant. Philos Trans R Soc Lond B Biol Sci 369:20130221. https://doi.org/10.1098/rstb.2013.0221

Dall'Osto L, Cazzaniga S, Bressan M et al (2017) Two mechanisms for dissipation of excess light in monomeric and trimeric light-harvesting complexes. Nat Plants 3:17033. https://doi.org/10.1038/ nplants.2017.33

de Bianchi S, Betterle N, Kouril R et al (2011) Arabidopsis mutants deleted in the light-harvesting protein Lhcb4 have a disrupted photosystem II macrostructure and are defective in photoprotection. Plant Cell 23:2659-2679. https://doi.org/10.1105/tpc.111.087320

Dominici P, Caffarri S, Armenante F et al (2002) Biochemical properties of the PsbS subunit of photosystem II either purified from chloroplast or recombinant. J Biol Chem 277:22750-22758. https ://doi.org/10.1074/jbc.M200604200

Elrad D, Niyogi KK, Grossman AR (2002) A major light-harvesting polypeptide of photosystem II functions in thermal dissipation. Plant Cell 14:1801-1816. https://doi.org/10.1105/tpc.002154

Emanuelsson O, Nielsen H, Von Heijne G (1999) ChloroP, a neural network-based method for predicting chloroplast transit peptides and their cleavage sites. Protein Sci 8:978-984

Fan M, Li M, Liu Z et al (2015) Crystal structures of the PsbS protein essential for photoprotection in plants. Nat Struct Mol Biol 22:729-735. https://doi.org/10.1038/nsmb.3068

Ferrante P, Ballottari M, Bonente G et al (2012) LHCBM1 and LHCBM2/7 polypeptides, components of major LHCII complex, have distinct functional roles in photosynthetic antenna system of Chlamydomonas reinhardtii. J Biol Chem 287:16276-16288. https://doi.org/10.1074/jbc.M111.316729

Genty B, Harbinson J, Briantais JM, Baker NR (1990) The relationship between non-photochemical quenching of chlorophyll fluorescence and the rate of photosystem 2 photochemistry in leaves. Photosynth Res 25:249-257. https://doi.org/10.1007/BF00033166 
Gerotto C, Alboresi A, Giacometti GM et al (2012) Coexistence of plant and algal energy dissipation mechanisms in the moss Physcomitrella patens. New Phytol 196:763-773. https://doi.org/10. 1111/j.1469-8137.2012.04345.x

Girolomoni L, Ferrante P, Berteotti S et al (2016) The function of LHCBM4/6/8 antenna proteins in Chlamydomonas reinhardtii. $\mathrm{J}$ Exp Bot 68:627-641. https://doi.org/10.1093/jxb/erw462

Horton P (2000) Prospects for crop improvement through the genetic manipulation of photosynthesis: morphological and biochemical aspects of light capture. J Exp Bot 51:475-485. https://doi. org/10.1093/jexbot/51.suppl_1.475

Horton P, Ruban AV, Walters RG (1996) Regulation of light harvesting in green plants. Annu Rev Plant Physiol Plant Mol Biol 47:655684. https://doi.org/10.1146/annurev.arplant.47.1.655

Kanazawa A, Kramer DM (2002) In vivo modulation of nonphotochemical exciton quenching (NPQ) by regulation of the chloroplast ATP synthase. Proc Natl Acad Sci USA 99:12789-12794. https://doi.org/10.1073/pnas.182427499

Karimi M, Inzé D, Depicker A (2002) GATEWAY vectors for Agrobacterium-mediated plant transformation. Trends Plant Sci 7:193-195

Kondo T, Pinnola A, Chen WJ et al (2017) Single-molecule spectroscopy of LHCSR1 protein dynamics identifies two distinct states responsible for multi-timescale photosynthetic photoprotection. Nat Chem 9:772

Kondo T, Gordon JB, Pinnola A et al (2019) Microsecond and millisecond dynamics in the photosynthetic protein LHCSR1 observed by single-molecule correlation spectroscopy. Proc Natl Acad Sci USA 116(23):11247-11252. https://doi.org/10.1073/pnas.18212 07116

Kramer DM, Sacksteder CA, Cruz JA (1999) How acidic is the lumen? Photosynth Res 60:151-163. https://doi.org/10.1023/A:10062 12014787

Li X-P, Björkman O, Shih C et al (2000) A pigment-binding protein essential for regulation of photosynthetic light harvesting. Nature 403:391-395. https://doi.org/10.1038/35000131

Li X-P, Gilmore AM, Caffarri S et al (2004) Regulation of photosynthetic light harvesting involves intrathylakoid lumen $\mathrm{pH}$ sensing by the PsbS protein. J Biol Chem 279:22866-22874. https://doi. org/10.1074/jbc.M402461200

Liguori N, Roy LM, Opacic M et al (2013) Regulation of light harvesting in the green alga Chlamydomonas reinhardtii: the c-terminus of lhesr is the knob of a dimmer switch. J Am Chem Soc 135:18339-18342. https://doi.org/10.1021/ja4107463

Morosinotto T, Baronio R, Bassi R (2002) Dynamics of chromophore binding to Lhe proteins in vivo and in vitro during operation of the xanthophyll cycle. J Biol Chem 277:36913-36920. https://doi. org/10.1074/jbc.M205339200

Morosinotto T, Segalla A, Giacometti GM, Bassi R (2010) Purification of structurally intact grana from plants thylakoids membranes. J Bioenerg Biomembr 42:37-45. https://doi.org/10.1007/s 1086 3-009-9261-3

Müller P, Li X-PP, Niyogi KK, Muller P (2001) Non-photochemical quenching. a response to excess light energy. Plant Physiol 125:1558-1566. https://doi.org/10.1104/pp.125.4.1558

Munekage Y, Hojo M, Meurer J et al (2002) PGR5 is involved in cyclic electron flow around photosystem $\mathrm{i}$ and is essential for photoprotection in Arabidopsis. Cell 110:361-371. https://doi.org/10.1016/ S0092-8674(02)00867-X

Munekage Y, Hashimoto M, Miyake C et al (2004) Cyclic electron flow around photosystem I is essential for photosynthesis. Nature 429:579-582. https://doi.org/10.1038/nature02598

Niyogi KK, Grossman AR, Björkman O (1998) Arabidopsis mutants define a central role for the Xanthophyll cycle in the regulation of photosynthetic energy conversion. Plant Cell 10:1121-1134
Peers G, Truong TB, Ostendorf E et al (2009) An ancient light-harvesting protein is critical for the regulation of algal photosynthesis. Nature 462:518-521

Peter GF, Takeuchi T, Philip Thornber J (1991) Solubilization and two-dimensional electrophoretic procedures for studying the organization and composition of photosynthetic membrane polypeptides. Methods 3:115-124. https://doi.org/10.1016/S1046 $-2023(05) 80203-8$

Pietrzykowska M, Suorsa M, Semchonok DA et al (2014) The lightharvesting chlorophyll $\mathrm{a} / \mathrm{b}$ binding proteins Lhcb1 and Lhcb2 play complementary roles during state transitions in Arabidopsis. Plant Cell 26:3646-3660. https://doi.org/10.1105/tpc.114.127373

Pinnola A, Dall'Osto L, Gerotto C et al (2013) Zeaxanthin binds to light-harvesting complex stress-related protein to enhance nonphotochemical quenching in Physcomitrella patens. Plant Cell 25:3519-3534. https://doi.org/10.1105/tpc.113.114538

Pinnola A, Cazzaniga S, Alboresi A et al (2015a) Light-harvesting complex stress-related proteins catalyze excess energy dissipation in both photosystems of Physcomitrella patens. Plant Cell 27:3213-3227. https://doi.org/10.1105/tpc.15.00443

Pinnola A, Ghin L, Gecchele E et al (2015b) Heterologous expression of moss light-harvesting complex stress-related 1 (LHCSR1), the chlorophyll a-xanthophyll pigment-protein complex catalyzing non-photochemical quenching, in Nicotiana sp. J Biol Chem 290:24340-24354. https://doi.org/10.1074/jbc.M115.668798

Pinnola A, Staleva-Musto H, Capaldi S et al (2016) Electron transfer between carotenoid and chlorophyll contributes to quenching in the LHCSR1 protein from Physcomitrella patens. Biochim Biophys Acta Bioenerg 1857:1870-1878. https://doi.org/10.1016/j. bbabio.2016.09.001

Pinnola A, Ballottari M, Bargigia I et al (2017) Functional modulation of LHCSR1 protein from Physcomitrella patens by zeaxanthin binding and low pH. Sci Rep 7:11158. https://doi.org/10.1038/ s41598-017-11101-7

Pogson B, McDonald KA, Truong M et al (1996) Arabidopsis carotenoid mutants demonstrate that lutein is not essential for photosynthesis in higher plants. Plant Cell 8:1627-1639. https://doi. org/10.1105/tpc.8.9.1627

Pribil M, Labs M, Leister D (2014) Structure and dynamics of thylakoids in land plants. J Exp Bot 65:1955-1972. https://doi. org/10.1093/jxb/eru090

Ruban AV, Phillip D, Young AJ, Horton P (1998) Excited-state energy level does not determine the differential effect of violaxanthin and zeaxanthin on chlorophyll fluorescence quenching in the isolated light-harvesting complex of photosystem II. Photochem Photobiol 68:829-834. https://doi.org/10.1111/j.1751-1097.1998.tb05291.x

Ruban AV, Berera R, Ilioaia C et al (2007) Identification of a mechanism of photoprotective energy dissipation in higher plants. Nature 450:575-578. https://doi.org/10.1038/nature06262

Zhang X, Henriques R, Lin S-S et al (2006) Agrobacterium-mediated transformation of Arabidopsis thaliana using the floral dip method. Nat Protoc 1:641-646. https://doi.org/10.1038/nprot .2006 .97

Publisher's Note Springer Nature remains neutral with regard to jurisdictional claims in published maps and institutional affiliations. 


\section{Affiliations}

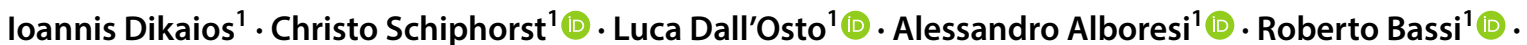
Alberta Pinnola ${ }^{1,2}$ (1)

Ioannis Dikaios

ioannisdikaios@hotmail.com

Christo Schiphorst

christo.schiphorst@univr.it

Luca Dall'Osto

luca.dallosto@univr.it

Alessandro Alboresi

alessandro.alboresi@unipd.it
Roberto Bassi

roberto.bassi@univr.it

1 Department of Biotechnology, University of Verona, Verona 37134, Italy

2 Department of Biology and Biotechnology, University of Pavia, Pavia 27100, Italy 